Federal Reserve Bank of Minneapolis

Research Department Staff Report 362

Revised December 2006

\title{
APPENDICES: Business Cycle Accounting ${ }^{\dagger}$
}

\author{
V.V. CHARI \\ University of Minnesota \\ and Federal Reserve Bank of Minneapolis \\ PATRICK J. KeHOE \\ Federal Reserve Bank of Minneapolis \\ and University of Minnesota \\ Ellen R. MCGRATtan \\ Federal Reserve Bank of Minneapolis \\ and University of Minnesota
}

$\dagger$ The views expressed herein are those of the authors and not necessarily those of the Federal Reserve Bank of Minneapolis or the Federal Reserve System. 


\section{Table of Contents}

A. Computation and Estimation . . . . . . . . . . . . . . . . . . . . . . 1

A.1. The Benchmark Model . . . . . . . . . . . . . . . . . . . . . . 1

A.2. Algorithms for Computing Equilibria . . . . . . . . . . . . . . . . 4

A.2.1. Nonlinear Computation . . . . . . . . . . . . . . . . . . . 5

A.2.2. Log-Linear Computation . . . . . . . . . . . . . . . . . . . 6

A.2.2.1. Checking a Test Case . . . . . . . . . . . . . . . . . 12

A.2.2.2. Computation for the General Log-Linear Case . . . . . . 13

A.2.3. Allowing for Adjustment Costs . . . . . . . . . . . . . . . . 13

A.2.4. An Alternative Investment Wedge . . . . . . . . . . . . . . 17

A.3. MLE Estimation . . . . . . . . . . . . . . . . . . . . . . . . . 18

A.3.1. State-Space Form . . . . . . . . . . . . . . . . . . . . . . 18

A.3.2. Log-Likelihood Function . . . . . . . . . . . . . . . . . . . 19

A.4. Decomposing Macro Aggregates . . . . . . . . . . . . . . . . . 20

A.4.1. Great Depression Period . . . . . . . . . . . . . . . . . . . 20

A.4.2. Postwar Period . . . . . . . . . . . . . . . . . . . . . . . 22

A.5. A Model with Capacity Utilization . . . . . . . . . . . . . . . . . 23

B. Data and Sources . . . . . . . . . . . . . . . . . . . . . . . . . . . 28

B.1. U.S. Historical Annual Data Measures and Sources . . . . . . . . . . 28

B.1.1. Measures . . . . . . . . . . . . . . . . . . . . . . . . . 28

B.1.2. Sources . . . . . . . . . . . . . . . . . . . . . . . . . . 29

B.1.3. Appendix Figures . . . . . . . . . . . . . . . . . . . . . . 33

B.2. U.S. Postwar Quarterly Data Measures and Sources . . . . . . . . . . 33

B.2.1. Measures . . . . . . . . . . . . . . . . . . . . . 33

B.2.2. Sources . . . . . . . . . . . . . . . . . . . . . . . . . . 34

C. Additional Material Not Reported in Text . . . . . . . . . . . . . . . . . 36 
C.1. Results for Government Consumption Wedge . . . . . . . . . . . . 36

C.2. Model with Maximum Investment Wedge . . . . . . . . . . . . . . 36

C.3. Wedges for the Capital Utilization Model . . . . . . . . . . . . . . 36

C.4. Results for an Alternative Investment Wedge . . . . . . . . . . . . 37

C.5. Proof of Propositions . . . . . . . . . . . . . . . . . . . . . . . 38

C.6. MLE Estimates for Alternative Models . . . . . . . . . . . . . . . 39 


\section{Appendix A. \\ Computation and Estimation}

In this Appendix, we describe the prototype model used in "Business Cycle Accounting" and the details of the computation of equilibria and estimation of parameters. We also discuss the sensitivity analysis we did for the benchmark prototype model allowing for variable capital utilization, investment adjustment costs, and an alternative specification for the investment wedge. Finally, we include figures, tables, and proofs not shown in the main text.

\section{A.1. The Benchmark Model}

Below we will use the following notation for our model variables:

$N$ : population $\left(N_{t}=\left(1+g_{n}\right)^{t}\right)$

c: per-capita consumption

$x$ : per-capita investment

$k$ : per-capita net capital stock

$l$ : per-capita labor input

tr: per-capita government transfers

$C$ : total consumption $\left(C_{t}=N_{t} c_{t}\right)$

$X$ : total investment

$K$ : total stock of capital

$L$ : total labor input in production

$Z$ : labor-augmenting technical change $\left(Z_{t}=z_{t}\left(1+g_{z}\right)^{t}\right)$

$r$ : rental rate on capital

$w$ : wage rate 
$\tau_{v}:$ tax rate on $v$

$\hat{v}$ : detrended per-capita variable $V$ (i.e., $\left.\hat{v}_{t}=V_{t} /\left[N_{t}\left(1+g_{z}\right)^{t}\right]\right)$

Consider an economy with households, firms, and the government. The representative household chooses consumption, investment, and labor to solve the following maximization problem:

$$
\max _{\left\{c_{t}, x_{t}, l_{t}\right\}} E \sum_{t=0}^{\infty} \beta^{t} U\left(c_{t}, 1-l_{t}\right) N_{t}
$$

subj. to $\left(1+\tau_{c t}\right) c_{t}+\left(1+\tau_{x t}\right) x_{t}=\left(1-\tau_{k t}\right) r_{t} k_{t}+\left(1-\tau_{l t}\right) w_{t} l_{t}+\tau_{k t} \delta k_{t}+\operatorname{tr}(A$

$$
\begin{aligned}
& N_{t+1} k_{t+1}=\left[(1-\delta) k_{t}+x_{t}\right] N_{t} \\
& c_{t}, x_{t} \geq 0 \quad \text { in all states, }
\end{aligned}
$$

taking processes for the rental rate, wage rate, the tax rates, and transfers as given. The representative firm solves a simple static problem at $t$ :

$$
\max _{\left\{K_{t}, L_{t}\right\}} F\left(K_{t}, Z_{t} L_{t}\right)-r_{t} K_{t}-w_{t} L_{t}
$$

The government sets rates of taxes and transfers in such a way that their budget constraint at $t$, namely,

$$
G_{t}+N_{t} t_{t}=\tau_{k t}\left(r_{t}-\delta\right) N_{t} k_{t}+\tau_{l t} w_{t} l_{t} N_{t}+\tau_{c t} N_{t} c_{t}+\tau_{x t} N_{t} x_{t}
$$

is satisfied. In equilibrium, the following conditions must hold:

$$
\begin{aligned}
& N_{t}\left(c_{t}+x_{t}\right)+G_{t}=F\left(K_{t}, Z_{t} L_{t}\right) \\
& N_{t} k_{t}=K_{t} \\
& N_{t} l_{t}=L_{t} .
\end{aligned}
$$


We now derive first-order conditions in this economy. The Lagrangian for the household optimization problem is given by

$$
\begin{aligned}
\mathcal{L}= & E \sum_{t} \tilde{\beta}^{t}\left\{U\left(\hat{c}_{t}, 1-l_{t}\right)+\frac{\zeta}{3} \min \left(\hat{x}_{t}, 0\right)^{3}\right. \\
& +\mu_{t}\left\{\left(1-\tau_{k t}\right) r_{t} \hat{k}_{t}+\left(1-\tau_{l t}\right) \hat{w}_{t} l_{t}+\tau_{k t} \delta \hat{k}_{t}+\hat{t}_{t}-\left(1+\tau_{c t}\right) \hat{c}_{t}-\left(1+\tau_{x t}\right) \hat{x}_{t}\right\} \\
& \left.+\lambda_{t}\left\{(1-\delta) \hat{k}_{t}+\hat{x}_{t}-\left(1+g_{z}\right)\left(1+g_{n}\right) \hat{k}_{t+1}\right\}\right\},
\end{aligned}
$$

where $\tilde{\beta}=\beta\left(1+g_{n}\right) h\left(1+g_{z}\right)$ and $h(\cdot)$ depends on our choice of utility. If $U(c, 1-l)=$ $c^{1-\sigma} v(l)$, then $h\left(1+g_{z}\right)=\left(1+g_{z}\right)^{1-\sigma}$. Notice that the Lagrangian has no term for the nonnegativity constraint on investment. Instead, we have included a penalty function indexed by $\zeta$. As $\zeta$ approaches infinity, the solution to the problem with a penalty function and no constraint on investment is the same as the solution to the original problem with $\zeta=0$ and $x_{t} \geq 0$ imposed.

The first-order conditions for the problem are

$$
\begin{gathered}
\frac{U_{2}\left(\hat{c}_{t}, 1-l_{t}\right)}{U_{1}\left(\hat{c}_{t}, 1-l_{t}\right)}=\frac{1-\tau_{l t}}{1+\tau_{c t}} \hat{w}_{t} \\
\frac{1+\tau_{x t}}{1+\tau_{c t}} U_{1}\left(\hat{c}_{t}, 1-l_{t}\right)-\zeta \min \left(\hat{x}_{t}, 0\right)^{2} \\
=\hat{\beta} E_{t}\left[\frac{U_{1}\left(\hat{c}_{t+1}, 1-l_{t+1}\right)}{1+\tau_{c t+1}}\right. \\
\left\{\left(1-\tau_{k t+1}\right) r_{t+1}+\delta \tau_{k t+1}+(1-\delta)\left(1+\tau_{x t+1}\right)\right\} \\
\left.-(1-\delta) \zeta \min \left(\hat{x}_{t+1}, 0\right)^{2}\right],
\end{gathered}
$$

where $\hat{\beta}=\beta h\left(1+g_{z}\right) /\left(1+g_{z}\right)$. If $U(c, l)=c^{1-\sigma} v(l)$, then $\hat{\beta}=\beta\left(1+g_{z}\right)^{-\sigma}$.

In addition, we have first-order conditions for the firm's static problem. These are

$$
\begin{aligned}
r_{t} & =F_{1}\left(\hat{k}_{t}, z_{t} l_{t}\right) \\
\hat{w}_{t} & =F_{2}\left(\hat{k}_{t}, z_{t} l_{t}\right) z_{t} .
\end{aligned}
$$


Finally, we have a resource constraint given by

$$
\hat{c}_{t}+\hat{g}_{t}+\hat{x}_{t}=F\left(\hat{k}_{t}, z_{t} l_{t}\right)
$$

once we detrend variables.

\section{A.2. Algorithms for Computing Equilibria}

Below we show how to compute the equilibrium using a nonlinear method and a log-linear method. We need to use the nonlinear methods to compute equilibrium paths during the 1930s because the declines in aggregate variables are so large. We use the log-linear method when we derive estimates of the process for the wedges and for computing equilibria in the postwar period. The log-linear method is convenient with our maximum likelihood estimation (MLE) procedure because a nonlinear method with a very large state space is computationally demanding when computing likelihood estimates. ${ }^{1}$ With estimates of our stochastic process we determine expectations. These expectations are inputs to our nonlinear model and used when we do our accounting exercise.

From here on, we make the following functional form assumptions and auxiliary choices:

$$
\begin{aligned}
& F(k, l)=k^{\theta} l^{1-\theta} \\
& U(c, 1-l)=\left(c(1-l)^{\psi}\right)^{1-\sigma} /(1-\sigma) \\
& s_{t+1}=P_{0}+P s_{t}+Q \epsilon_{s, t+1}, \quad \epsilon_{s} \sim N(0, I) \text { (which is represented eith } \\
& \text { chain using the method of Tauchen } 1986 \text { or as a continuous process). } \\
& \log \left(z_{t}\right)=\log z\left(s_{t}\right) \\
& \log \hat{g}_{t}=\log \hat{g}\left(s_{t}\right) \\
& \tau_{l t}=\tau_{l}\left(s_{t}\right)
\end{aligned}
$$$$
s_{t+1}=P_{0}+P s_{t}+Q \epsilon_{s, t+1}, \quad \epsilon_{s} \sim N(0, I) \text { (which is represented either as a Markov }
$$

1 We experimented with very different expectations and found that they had only a very small impact on our results and, therefore, did not affect our conclusions. 


$$
\begin{aligned}
& \tau_{x t}=\tau_{x}\left(s_{t}\right) \\
& \tau_{k t}=\tau_{k}\left(s_{t}\right) \\
& \tau_{c t}=\tau_{c}\left(s_{t}\right)
\end{aligned}
$$

\section{A.2.1. Nonlinear Computation}

For our nonlinear solution method, we assume that the vector autoregressive process for the state can be well approximated by a Markov chain. Let $s$ be the index for the state. Then at time $t$, if the state is $s, \hat{g}_{t}=\hat{g}(s), \tau_{l t}=\tau_{l}(s), \tau_{x t}=\tau_{x}(s)$, and $z_{t}=z(s)$. The transition matrix for $s$ is given by $\Pi$ with $\pi\left(s, s^{\prime}\right)$ being the probability of going from state $s$ to state $s^{\prime}$.

The state of the economy in any period can be summarized by two scalars: $\hat{k}$ and $s$. Our Fortran code computes the decision rule $\hat{c}(\hat{k}, s)$. All other decisions can be determined

via static first-order conditions once we know $\hat{c}(\hat{k}, s)$. In particular, $l(\hat{k}, s)$ and $\hat{x}(\hat{k}, s)$ can be determined once we know consumption.

To compute $\hat{c}(\hat{k}, s)$, we apply the finite-element method using the dynamic first-order condition as the residual and Galerkin bases. More specifically, we assume that the consumption function is well approximated by

$$
\hat{c}(\hat{k}, s)=\sum_{j=1}^{\text {nnodes }} \alpha_{j}^{s} \Psi_{j}(k)
$$

where the $\Psi_{j}$ is a function that takes on nonzero values in two cells (or "elements") of a grid over $\hat{k}$ around grid point (or "node") $j$. The algorithm is to find the coefficients $\alpha_{j}^{s}$, $j=1, \ldots$ nnodes, $s=1, \ldots S$ that satisfy the following equations:

$$
\int R(\hat{k}, s ; \alpha) \Psi_{j}(\hat{k}) d \hat{k}=0
$$


for all $s$ and $j$ where

$$
\begin{aligned}
R(\hat{k}, s ; \alpha)= & \frac{1+\tau_{x}(s)}{1+\tau_{c}(s)} U_{1}(\hat{c}, 1-l) \\
& -\zeta \min (\hat{x}, 0)^{2}+\hat{\beta}(1-\delta) \zeta \sum_{s^{\prime}} \pi_{s, s^{\prime}} \min \left(\hat{x}^{\prime}, 0\right)^{2} \\
& -\hat{\beta} \sum_{s^{\prime}} \pi_{s, s^{\prime}} \frac{U_{1}\left(\hat{c}^{\prime}, 1-l^{\prime}\right)}{1+\tau_{c}\left(s^{\prime}\right)} \\
& \quad\left\{\left(1-\tau_{k}\left(s^{\prime}\right)\right) F_{1}\left(\hat{k}^{\prime}, z\left(s^{\prime}\right) l^{\prime}\right)+\tau_{k}\left(s^{\prime}\right) \delta+(1-\delta)\left(1+\tau_{x}(s)\right)\right\} .
\end{aligned}
$$

The investments $x$ and $x^{\prime}$ satisfy resource constraints

$$
\begin{aligned}
& \hat{x}=F(\hat{k}, z(s) l)-\sum_{j} \alpha_{j}^{s} \Psi_{j}(\hat{k})-\hat{g}(s) \\
& \hat{x}^{\prime}=F\left(\hat{k}^{\prime}, z\left(s^{\prime}\right) l^{\prime}\right)-\sum_{j} \alpha_{j}^{s} \Psi_{j}\left(\hat{k}^{\prime}\right)-\hat{g}\left(s^{\prime}\right) .
\end{aligned}
$$

The next period capital stock is given by

$$
\hat{k}^{\prime}=((1-\delta) \hat{k}+\hat{x}) /\left[\left(1+g_{n}\right)\left(1+g_{z}\right)\right] .
$$

The labor inputs $l$ and $l^{\prime}$ solve

$$
\begin{aligned}
& \frac{U_{2}(\hat{c}, 1-l)}{U_{1}(\hat{c}, 1-l)}=\frac{1-\tau_{l}(s)}{1+\tau_{c}(s)} F_{2}(\hat{k}, z(s) l) z(s) \\
& \frac{U_{2}\left(\hat{c}^{\prime}, 1-l^{\prime}\right)}{U_{1}\left(\hat{c}^{\prime}, 1-l^{\prime}\right)}=\frac{1-\tau_{l}\left(s^{\prime}\right)}{1+\tau_{c}\left(s^{\prime}\right)} F_{2}\left(\hat{k}^{\prime}, z\left(s^{\prime}\right) l^{\prime}\right) z\left(s^{\prime}\right) .
\end{aligned}
$$

\section{A.2.2. Log-Linear Computation}

We now describe the steps taken for the log-linear solution method (with an interior solution and $\zeta=0$ ). Because we are going to apply maximum likelihood estimation, we will derive the solution analytically. 
We start by writing the system of equations in terms of $k$ and $s$. This is done by replacing $r, w, \hat{c}$, and $\hat{x}$ in the first-order conditions with functions of the states. Thus we start with

$$
\begin{aligned}
& \hat{c}_{t}+\hat{g}_{t}+\left(1+g_{z}\right)\left(1+g_{n}\right) \hat{k}_{t+1}-(1-\delta) \hat{k}_{t}=\hat{y}_{t}=\hat{k}_{t}^{\theta}\left(z_{t} l_{t}\right)^{1-\theta} \\
& \frac{\psi \hat{c}_{t}}{1-l_{t}}=\left(1-\tau_{l t}\right)(1-\theta) \hat{k}_{t}^{\theta} l_{t}^{-\theta} z_{t}^{1-\theta} \\
& \left(1+\tau_{x t}\right) \hat{c}_{t}^{-\sigma}\left(1-l_{t}\right)^{\psi(1-\sigma)} \\
& \quad=\hat{\beta} E_{t} \hat{c}_{t+1}^{-\sigma}\left(1-l_{t+1}\right)^{\psi(1-\sigma)}\left[\theta \hat{k}_{t+1}^{\theta-1}\left(z_{t+1} l_{t+1}\right)^{1-\theta}+(1-\delta)\left(1+\tau_{x t+1}\right)\right]
\end{aligned}
$$

which can be reduced to the following:

$$
\begin{gathered}
\psi\left[\hat{k}_{t}^{\theta}\left(z_{t} l_{t}\right)^{1-\theta}-\left(1+g_{n}\right)\left(1+g_{z}\right) \hat{k}_{t+1}+(1-\delta) \hat{k}_{t}-\hat{g}_{t}\right] \\
=\left(1-\tau_{l t}\right)(1-\theta) \hat{k}_{t}^{\theta} l_{t}^{-\theta} z_{t}^{1-\theta}\left(1-l_{t}\right) \\
\left(1+\tau_{x t}\right)\left[\hat{k}_{t}^{\theta}\left(z_{t} l_{t}\right)^{1-\theta}-\left(1+g_{n}\right)\left(1+g_{z}\right) \hat{k}_{t+1}+(1-\delta) \hat{k}_{t}-\hat{g}_{t}\right]^{-\sigma}\left(1-l_{t}\right)^{\psi(1-\sigma)} \\
=\hat{\beta} E_{t}\left[\hat{k}_{t+1}^{\theta}\left(z_{t+1} l_{t+1}\right)^{1-\theta}-\left(1+g_{n}\right)\left(1+g_{z}\right) \hat{k}_{t+2}\right. \\
\left.+(1-\delta) \hat{k}_{t+1}-\hat{g}_{t+1}\right]^{-\sigma}\left(1-l_{t+1}\right)^{\psi(1-\sigma)} \\
{\left[\theta \hat{k}_{t+1}^{\theta-1}\left(z_{t+1} l_{t+1}\right)^{1-\theta}+(1-\delta)\left(1+\tau_{x t+1}\right)\right] .}
\end{gathered}
$$

Next, we compute the steady state of the system for constant values for $z$, the taxes, and government spending:

$$
\begin{aligned}
& \hat{k} / l=\left(\frac{\left(1+\tau_{x}\right)(1-\hat{\beta}(1-\delta))}{\hat{\beta} \theta z^{1-\theta}}\right)^{1 /(\theta-1)} \\
& \hat{c}=\left[(\hat{k} / l)^{\theta-1} z^{1-\theta}-\left(1+g_{z}\right)\left(1+g_{n}\right)+1-\delta\right] \hat{k}-\hat{g}=\xi_{1} \hat{k}-\hat{g} \\
& \hat{c}=\left[\left(1-\tau_{l}\right)(1-\theta)(\hat{k} / l)^{\theta} z^{1-\theta} / \psi\right](1-1 /(\hat{k} / l) \hat{k})=\xi_{2}-\xi_{3} \hat{k},
\end{aligned}
$$

where the last two equations imply $\hat{k}=\left(\xi_{2}+\hat{g}\right) /\left(\xi_{1}+\xi_{3}\right), \hat{c}=\xi_{1} \hat{k}-\hat{g}, l=(1 /(\hat{k} / l)) \hat{k}$.

The log-linearization is done around these steady-state values. Detrended consump- 
tion is given approximately by

$$
\begin{aligned}
\hat{c}_{t} \approx & \hat{c} \log \hat{c}_{t} \\
\approx & \hat{k}^{\theta}(z l)^{1-\theta}\left[\theta \log \hat{k}_{t}+(1-\theta)\left(\log z_{t}+\log l_{t}\right)\right] \\
& -\left(1+g_{z}\right)\left(1+g_{n}\right) \hat{k} \log \hat{k}_{t+1}+(1-\delta) \hat{k} \log \hat{k}_{t}-\hat{g} \log \hat{g}_{t} .
\end{aligned}
$$

The labor input is then derived from the static first-order condition $(A .2 .2)$ :

$$
\begin{aligned}
& 0 \approx \psi\left\{\hat{k}^{\theta}(z l)^{1-\theta}\left[\theta \log \hat{k}_{t}+(1-\theta)\left(\log z_{t}+\log l_{t}\right)\right]\right. \\
&\left.-\left(1+g_{z}\right)\left(1+g_{n}\right) \hat{k} \log \hat{k}_{t+1}+(1-\delta) \hat{k} \log \hat{k}_{t}-\hat{g} \log \hat{g}_{t}\right\} \\
&+(1-\theta)\left(1-\tau_{l}\right) \hat{k}^{\theta} l^{-\theta} z^{1-\theta}(1-l)\left\{1 /\left(1-\tau_{l}\right) \tau_{l t}\right. \\
&\left.\quad-\theta \log \hat{k}_{t}+\theta \log l_{t}-(1-\theta) \log z_{t}+l /(1-l) \log l_{t}\right\}
\end{aligned}
$$

which we write succinctly as

$$
\log l_{t}=\phi_{l k} \log \hat{k}_{t}+\phi_{l z} \log z_{t}+\phi_{l l} \tau_{l t}+\phi_{l g} \log \hat{g}_{t}+\phi_{l k^{\prime}} \log \hat{k}_{t+1}
$$

Using this equation for $\log l$, we use the other static first-order conditions to write $\log \hat{y}$, $\log \hat{x}$, and $\log \hat{c}$ as follows:

$$
\begin{aligned}
\log \hat{y}_{t}= & \phi_{y k} \log \hat{k}_{t}+\phi_{y z} \log z_{t}+\phi_{y l} \tau_{l t}+\phi_{y g} \log \hat{g}_{t}+\phi_{y k^{\prime}} \log \hat{k}_{t+1} \\
= & \left(\theta+(1-\theta) \phi_{l k}\right) \log \hat{k}_{t}+(1-\theta)\left(1+\phi_{l z}\right) \log z_{t} \\
& +(1-\theta)\left[\phi_{l l} \tau_{l t}+\phi_{l k^{\prime}} \log \hat{k}_{t+1}\right] \\
\log \hat{x}_{t}= & \left(1+g_{z}\right)\left(1+g_{n}\right) \hat{k} / \hat{x} \log \hat{k}_{t+1}-(1-\delta) \hat{k} / \hat{x} \log \hat{k}_{t} \\
\log \hat{c}_{t}= & \phi_{c k} \log \hat{k}_{t}+\phi_{c z} \log z_{t}+\phi_{c l} \tau_{l t}+\phi_{c g} \log \hat{g}_{t}+\phi_{c k^{\prime}} \log \hat{k}_{t+1} \\
= & {\left[\hat{y} \log y_{t}-\hat{x} \log x_{t}-\hat{g} \log \hat{g}_{t}\right] / \hat{c} }
\end{aligned}
$$

where the $\phi$ 's are known functions of the parameters.

Capital is derived from the dynamic first-order condition

$$
0 \approx\left(1+\tau_{x}\right) \hat{c}^{-\sigma}(1-l)^{\psi(1-\sigma)}\left\{-\psi(1-\sigma) l /(1-l) \log l_{t}-\sigma \log \hat{c}_{t}\right\}
$$




$$
\begin{gathered}
\quad+\hat{c}^{-\sigma}(1-l)^{\psi(1-\sigma)} \tau_{x t} \\
-\hat{\beta} E_{t}\left\{\left[\theta \hat{k}^{\theta-1}(z l)^{1-\theta}+(1-\delta)\left(1+\tau_{x}\right)\right]\right. \\
\cdot\left[\hat{c}^{-\sigma}(1-l)^{\psi(1-\sigma)}\left\{-\psi(1-\sigma) l /(1-l) \log l_{t+1}-\sigma \log \hat{c}_{t+1}\right\}\right] \\
+\hat{c}^{-\sigma}(1-l)^{\psi(1-\sigma)}\left[\theta \hat{k}^{\theta-1}(z l)^{1-\theta}(1-\theta)\right. \\
\left.\left.\quad \cdot\left(\log l_{t+1}+\log z_{t+1}-\log \hat{k}_{t+1}\right)+(1-\delta) \tau_{x t+1}\right]\right\}
\end{gathered}
$$

which simplifies to

$$
\begin{aligned}
0 \approx(1+ & \left.\tau_{x}\right)\left\{-\psi(1-\sigma) l /(1-l) \log l_{t}-\sigma \log \hat{c}_{t}\right\}+\tau_{x t} \\
- & E_{t}\left\{\left(1+\tau_{x}\right)\left\{-\psi(1-\sigma) l /(1-l) \log l_{t+1}-\sigma \log \hat{c}_{t+1}\right\}\right. \\
& \left.\quad+\hat{\beta}\left[r(1-\theta)\left(\log l_{t+1}+\log z_{t+1}-\log \hat{k}_{t+1}\right)+(1-\delta) \tau_{x t+1}\right]\right\}
\end{aligned}
$$

where $r=\theta \hat{y} / \hat{k}$.

We guess the following form of the solution for capital:

$$
\log \left(\hat{k}_{t+1}\right)=\gamma_{0}+\gamma_{k} \log \hat{k}_{t}+\gamma_{z} \log z_{t}+\gamma_{l} \tau_{l t}+\gamma_{x} \tau_{x t}+\gamma_{g} \log \hat{g}_{t}
$$

and set $\gamma$ 's so that the dynamic residual $(A .2 .8)$ is exactly 0 . We can do this by first ignoring shock terms and find $\gamma_{k}$ that satisfies a particular quadratic equation (that does not depend on any other $\gamma$ coefficient). Then, we can find $\gamma_{z}, \gamma_{l}, \gamma_{x}$, and $\gamma_{g}$ by solving a linear system of equations with $\gamma_{k}$ assumed known. Finally, we use the steady-state equations to determine $\gamma_{0}$.

We start by deriving $\gamma_{k}$. To do this, we need to write out the coefficients on $\hat{k}_{t+2}, \hat{k}_{t+1}$, and $\hat{k}_{t}$ in the dynamic first-order condition $(A .2 .8)$ making use of the $\phi$ 's from the static first-order conditions $(A .2 .4)-(A .2 .7)$. For now, we can ignore the expectations operator. 
We get the following:

$$
\begin{gathered}
0 \approx-\left(1+\tau_{x}\right)\left\{-\psi(1-\sigma) l /(1-l)\left[\phi_{l k} \log \hat{k}_{t}+\phi_{l k^{\prime}} \log \hat{k}_{t+1}\right]\right. \\
\left.-\sigma\left[\phi_{c k} \log \hat{k}_{t}+\phi_{c k^{\prime}} \log \hat{k}_{t+1}\right]\right\} \\
+E_{t}\left(1+\tau_{x}\right)\left\{-\psi(1-\sigma) l /(1-l)\left[\phi_{l k} \log \hat{k}_{t+1}+\phi_{l k^{\prime}} \log \hat{k}_{t+2}\right]\right. \\
\left.-\sigma\left[\phi_{c k} \log \hat{k}_{t+1}+\phi_{c k^{\prime}} \log \hat{k}_{t+2}\right]\right\} \\
+\hat{\beta} E_{t}\left[r(1-\theta)\left(\left(\phi_{l k}-1\right) \log \hat{k}_{t+1}+\phi_{l k^{\prime}} \hat{k}_{t+2}\right)\right]
\end{gathered}
$$

which simplifies to

$$
\begin{aligned}
& 0=\left[\hat{\beta} r(1-\theta) \phi_{l k^{\prime}}-\left(1+\tau_{x}\right) \psi(1-\sigma) l /(1-l) \phi_{l k^{\prime}}-\left(1+\tau_{x}\right) \sigma \phi_{c k^{\prime}}\right] \hat{k}_{t+2} \\
& +\left[\hat{\beta} r(1-\theta)\left(\phi_{l k}-1\right)-\left(1+\tau_{x}\right) \psi(1-\sigma) l /(1-l) \phi_{l k}-\left(1+\tau_{x}\right) \sigma \phi_{c k}\right. \\
& \left.+\left(1+\tau_{x}\right) \psi(1-\sigma) l /(1-l) \phi_{l k^{\prime}}+\left(1+\tau_{x}\right) \sigma \phi_{c k^{\prime}}\right] \hat{k}_{t+1} \\
& -\left[-\left(1+\tau_{x}\right) \psi(1-\sigma) l /(1-l) \phi_{l k}-\left(1+\tau_{x}\right) \sigma \phi_{c k}\right] \hat{k}_{t} \\
& + \text { all other terms }
\end{aligned}
$$

or, more succinctly, rewrite $(A .2 .10)$ as $\left(a+b L+c L^{2}\right) \hat{k}_{t+2}=$ other terms, where $\gamma_{k}$ is the root of the quadratic inside the unit circle. Note that $\gamma_{k}$ does not depend on the other unknown $\gamma$ 's.

Given $\gamma_{k}$, we can solve a linear system for the other $\gamma^{\prime}$ s. At this point, we do not ignore the expectations operator:

$$
\begin{gathered}
0 \approx\left(1+\tau_{x}\right)\left\{-\psi(1-\sigma) l /(1-l)\left[\left(\phi_{l z}+\phi_{l k^{\prime}} \gamma_{z}\right) \log z_{t}\right.\right. \\
+\left(\phi_{l l}+\phi_{l k^{\prime}} \gamma_{l}\right) \tau_{l t} \\
+\phi_{l k^{\prime}} \gamma_{x} \tau_{x t}+\left(\phi_{l g}+\phi_{l k^{\prime}} \gamma_{g}\right) \log \hat{g}_{t} \\
-\sigma\left[\left(\phi_{c z}+\phi_{c k^{\prime}} \gamma_{z}\right) \log z_{t}+\left(\phi_{c l}+\phi_{c k^{\prime}} \gamma_{l}\right) \tau_{l t}\right. \\
\left.\left.+\phi_{c k^{\prime}} \gamma_{x} \tau_{x t}+\left(\phi_{c g}+\phi_{c k^{\prime}} \gamma_{l}\right) \log \hat{g}_{t}\right]\right\}+\tau_{x t}
\end{gathered}
$$




$$
\begin{aligned}
& -E_{t}\left(1+\tau_{x}\right)\left\{-\psi(1-\sigma) l /(1-l)\left[\left(\phi_{l k}+\phi_{l k^{\prime}} \gamma_{k}\right)\left(\gamma_{z} \log z_{t}+\gamma_{l} \tau_{l t}+\gamma_{x} \tau_{x t}+\gamma_{g} \log \hat{g}_{t}\right)\right.\right. \\
& +\left(\phi_{l z}+\phi_{l k^{\prime}} \gamma_{z}\right) \log z_{t+1} \\
& +\left(\phi_{l l}+\phi_{l k^{\prime}} \gamma_{l}\right) \tau_{l t+1} \\
& +\phi_{l k^{\prime}} \gamma_{x} \tau_{x t+1} \\
& \left.+\left(\phi_{l g}+\phi_{l k^{\prime}} \gamma_{g}\right) \log \hat{g}_{t+1}\right] \\
& -\sigma\left[\left(\phi_{c k}+\phi_{c k^{\prime}} \gamma_{k}\right)\left(\gamma_{z} \log z_{t}+\gamma_{l} \tau_{l t}+\gamma_{x} \tau_{x t}+\gamma_{g} \log \hat{g}_{t}\right)\right. \\
& +\left(\phi_{c z}+\phi_{c k^{\prime}} \gamma_{z}\right) \log z_{t+1} \\
& +\left(\phi_{c l}+\phi_{c k^{\prime}} \gamma_{l}\right) \tau_{l t+1} \\
& +\phi_{c k^{\prime}} \gamma_{x} \tau_{x t+1} \\
& \left.\left.+\left(\phi_{c g}+\phi_{c k^{\prime}} \gamma_{g}\right) \log \hat{g}_{t+1}\right]\right\} \\
& -\hat{\beta} r(1-\theta) E_{t}\left[\left(\phi_{l k}+\phi_{l k^{\prime}} \gamma_{k}-1\right)\left(\gamma_{z} \log z_{t}+\gamma_{l} \tau_{l t}+\gamma_{x} \tau_{x t}+\gamma_{g} \log \hat{g}_{t}\right)\right. \\
& +\left(\phi_{l z}+\phi_{l k^{\prime}} \gamma_{z}+1\right) \log z_{t+1} \\
& +\left(\phi_{l l}+\phi_{l k^{\prime}} \gamma_{l}\right) \tau_{l t+1} \\
& +\phi_{l k^{\prime}} \gamma_{x} \tau_{x t+1} \\
& \left.+\left(\phi_{l g}+\phi_{l k^{\prime}} \gamma_{g}\right) \log \hat{g}_{t+1}\right] \\
& -\hat{\beta}(1-\delta) E_{t} \tau_{x t+1},
\end{aligned}
$$

which reduces to

$$
\begin{aligned}
0 & =\left(\kappa_{0}+\kappa_{1} \cdot \gamma_{s}\right)^{\prime}\left[\log z_{t}, \tau_{l t}, \tau_{x t}, \log \hat{g}_{t}\right]^{\prime}+\left(\zeta_{0}+\zeta_{1} \cdot \gamma_{s}\right)^{\prime} E_{t}\left[\log z_{t+1}, \tau_{l t+1}, \tau_{x t+1}, \log \hat{g}_{t+1}\right]^{\prime} \\
& =\left(\kappa_{0}+\kappa_{1} \cdot \gamma_{s}\right)^{\prime}\left[\log z_{t}, \tau_{l t}, \tau_{x t}, \log \hat{g}_{t}\right]^{\prime}+\left(\zeta_{0}+\zeta_{1} \cdot \gamma_{s}\right)^{\prime} P\left[\log z_{t}, \tau_{l t}, \tau_{x t}, \log \hat{g}_{t}\right]^{\prime}, \quad(A .2 .11)
\end{aligned}
$$

where $x \cdot y$ denotes element-by-element multiplication of vectors $x$ and $y ; \kappa_{0}, \kappa_{1}, \zeta_{0}$, and $\zeta_{1}$ are vectors of length 4 with elements equal to functions of the parameters and $\gamma_{k}$; and $\gamma_{s}=\left[\gamma_{z}, \gamma_{l}, \gamma_{x}, \gamma_{g}\right]^{\prime}$. The $\gamma^{\prime}$ s that set this residual to zero satisfy a four-dimensional linear system,

$$
\left(\kappa_{0}+\kappa_{1} \cdot \gamma_{s}\right)^{\prime}+\left(\zeta_{0}+\zeta_{1} \cdot \gamma_{s}\right)^{\prime} a=0
$$




\section{A.2.2.1. Checking a Test Case}

Assume $g_{n}=g_{z}=\psi=0, \sigma=\delta=1, \hat{g}_{t}=\tau_{l t}=\tau_{x t}=0$, and

$$
\log z_{t+1}=\rho_{0}+\rho \log z_{t}+\epsilon_{z, t+1}
$$

so that

$$
P=\left[\begin{array}{ccccc}
\rho_{z} & 0 & 0 & 0 & \rho_{0} \\
0 & 0 & 0 & 0 & 0 \\
0 & 0 & 0 & 0 & 0 \\
0 & 0 & 0 & 0 & 0 \\
0 & 0 & 0 & 0 & 1
\end{array}\right], \quad Q=\left[\begin{array}{cccc}
\sigma_{z} & 0 & 0 & 0 \\
0 & 0 & 0 & 0 \\
0 & 0 & 0 & 0 \\
0 & 0 & 0 & 0 \\
0 & 0 & 0 & 0
\end{array}\right]
$$

The solution in this case:

$$
\log \hat{k}_{t+1}=\log (\beta \theta)+\theta \log \hat{k}_{t}+(1-\theta) \log z_{t}
$$

Using the formulas above, we have the following steady state:

$$
\begin{aligned}
& \hat{k}=(\beta \theta)^{1 /(1-\theta)} z \\
& \hat{c}=(1-\beta \theta) /(\beta \theta) \hat{k} \\
& \hat{y}=z^{1-\theta} \hat{k}^{\theta}
\end{aligned}
$$

and $\gamma_{k}$ from quadratic

$$
\begin{aligned}
0 & =-\phi_{c k^{\prime}} \hat{k}_{t+2}-\left[\hat{\beta} r(1-\theta)+\phi_{c k}-\phi_{c k^{\prime}}\right] \hat{k}_{t+1}+\phi_{c k} \hat{k}_{t}+\text { all other terms } \\
& =\beta \theta /(1-\beta \theta) \hat{k}_{t+2}-[(1-\theta)+\theta /(1-\beta \theta)-\beta \theta /(1-\beta \theta)] \hat{k}_{t+1}+[\theta /(1-\beta \theta)] \hat{k}_{t} \\
& =\beta \theta \hat{k}_{t+2}-[(1-\theta)(1-\beta \theta)+\theta-\beta \theta] \hat{k}_{t+1}+\theta \hat{k}_{t},
\end{aligned}
$$

which implies $\gamma_{k}=\theta$. The other coefficients satisfy

$$
\begin{aligned}
0 \approx- & \left(\phi_{c z}+\phi_{c k^{\prime}} \gamma_{z}\right) \log z_{t}-\phi_{c k^{\prime}} \gamma_{l} \tau_{l t}-\phi_{c k^{\prime}} \gamma_{x} \tau_{x t}-\phi_{c k^{\prime}} \gamma_{g} \log \hat{g}_{t}+\tau_{x t} \\
+\left(\phi_{c k}\right. & \left.+\phi_{c k^{\prime}} \theta\right)\left(\gamma_{z} \log z_{t}+\gamma_{l} \tau_{l t}+\gamma_{x} \tau_{x t}+\gamma_{g} \log \hat{g}_{t}\right) \\
& +\left(\phi_{c z}+\phi_{c k^{\prime}} \gamma_{z}\right) E_{t} \log z_{t+1} \\
& +(1-\theta)\left[\gamma_{z} \log z_{t}+\gamma_{l} \tau_{l t}+\gamma_{x} \tau_{x t}+\gamma_{g} \log \hat{g}_{t}-E_{t} \log z_{t+1}\right]
\end{aligned}
$$

and, therefore, $\gamma_{z}=1-\theta, \gamma_{l}=\gamma_{g}=0, \gamma_{x}=-1+\beta \theta$. 


\section{A.2.2.2. Computation for the General Log-Linear Case}

Assume that the solution is

$$
\log \hat{k}_{t+1}=a \log \hat{k}_{t}+b\left[\begin{array}{llll}
\log z_{t} & \tau_{l t} & \tau_{x t} & \log \hat{g}_{t}
\end{array}\right]^{\prime}+\text { constant }
$$

where $a$ is a scalar and $b$ is $1 \times 4$. Assume that the residual from the dynamic first-order condition is

$$
\begin{gathered}
f\left(E_{t} \log \hat{k}_{t+2}, \log \hat{k}_{t+1}, \log \hat{k}_{t}, \log z_{t+1}, \log z_{t}, \tau_{l t+1}, \tau_{l t}, \tau_{x t+1}, \tau_{x t}, \log \hat{g}_{t+1}, \log \hat{g}_{t}\right) \\
\approx a_{0} E_{t} \log \hat{k}_{t+2}+a_{1} \log \hat{k}_{t+1}+a_{2} \log \hat{k}_{t}+b_{0} E_{t} s_{t+1}+b_{1} s_{t} .
\end{gathered}
$$

Then the general solution algorithm is to find $a$ that solves the quadratic equation

$$
a_{0} a^{2}+a_{1} a+a_{2}=0
$$

and $b$ that solves the linear equations

$$
a_{0} a b+a_{0} b P+a_{1} b+b_{0} P+b_{1}=0_{1 \times 4} .
$$

Note that this implies

$$
b=-\left[\left(a_{0} a+a_{1}\right) I_{4 \times 4}+a_{0} P^{\prime}\right]^{-1}\left(b_{0} P+b_{1} I_{4 \times 4}\right)^{\prime} .
$$

\section{A.2.3. Allowing for Adjustment Costs}

To allow for adjustment costs, we solve the household maximization problem for the benchmark model, namely (A.1.1) subject to (A.1.2), (A.1.4), and

$$
N_{t+1} k_{t+1}=\left[(1-\delta) k_{t}+x_{t}-\varphi\left(x_{t} / k_{t}\right) k_{t}\right] N_{t}
$$

instead of (A.1.3), where

$$
\varphi(x / k)=\frac{a}{2}\left(\frac{x}{k}-b\right)^{2} .
$$


As a sensitivity check we set $b$ equal to the investment-capital trend rate (i.e., $b=(1+$ $\left.\left.g_{z}\right)\left(1+g_{n}\right)-1+\delta\right)$ and increase $a$ from 0 . To do this, we need to modify the dynamic first-order condition. We replace $(A .2 .8)$ by

$$
\begin{aligned}
{\left[\frac{1+\tau_{x t}}{1+\tau_{c t}} U_{1}\left(\hat{c}_{t}, 1-l_{t}\right)-\zeta \min \left(\hat{x}_{t}, 0\right)^{2}\right]\left(\frac{1}{1-\varphi^{\prime}\left(\hat{x}_{t} / \hat{k}_{t}\right)}\right) } \\
=\hat{\beta} E_{t}\left\{\frac{U_{1}\left(\hat{c}_{t+1}, 1-l_{t+1}\right)}{1+\tau_{c t+1}}\left\{\left(1-\tau_{k t+1}\right) r_{t+1}+\delta \tau_{k t+1}\right\}\right. \\
+\left[\frac{1+\tau_{x t+1}}{1+\tau_{c t+1}} U_{1}\left(\hat{c}_{t+1}, 1-l_{t+1}\right)-\zeta \min \left(\hat{x}_{t+1}, 0\right)^{2}\right] \\
\cdot\left(\frac{1}{1-\varphi^{\prime}\left(\hat{x}_{t+1} / \hat{k}_{t+1}\right)}\right) \\
\left.\cdot\left\{1-\delta-\varphi\left(\frac{\hat{x}_{t+1}}{\hat{k}_{t+1}}\right)+\varphi^{\prime}\left(\frac{\hat{x}_{t+1}}{\hat{k}_{t+1}}\right) \frac{\hat{x}_{t+1}}{\hat{k}_{t+1}}\right\}\right\} .
\end{aligned}
$$

In summary, allowing for adjustment costs involves two changes in the system of equations: replacing (A.1.3) and (A.2.8) with (A.2.12) and (A.2.13).

For the benchmark model with adjustment costs, we can construct

$$
\tilde{\tau}_{x t}=\frac{1+\tau_{x t}}{1-\varphi^{\prime}\left(\hat{x}_{t} / \hat{k}_{t}\right)}-1
$$

using series on $\tau_{x t}, \hat{x}_{t}$, and $\hat{k}_{t}$ from the benchmark model without adjustment costs. The effective investment wedge corresponding to this rate $\left(1 /\left(1+\tilde{\tau}_{x t}\right)\right)$, when fed into the benchmark model with adjustment costs, yields almost exactly the same results for equilibrium output, hours, and investment. (There is a slight difference because the effective depreciation rates are different for the two models.)

To do log-linear computation (as in the baseline economy) in the case with adjustment costs and $\tau_{c t}=\tau_{k t}=0$, we start with

$$
\hat{c}_{t}+\hat{g}_{t}+\left(1+g_{z}\right)\left(1+g_{n}\right) \hat{k}_{t+1}-(1-\delta) \hat{k}_{t}+\varphi\left(\hat{x}_{t} / \hat{k}_{t}\right) \hat{k}_{t}=\hat{y}_{t}=\hat{k}_{t}^{\theta}\left(z_{t} l_{t}\right)^{1-\theta}(A
$$




$$
\begin{gathered}
\frac{\psi \hat{c}_{t}}{1-l_{t}}=\left(1-\tau_{l t}\right)(1-\theta) \hat{k}_{t}^{\theta} l_{t}^{-\theta} z_{t}^{1-\theta} \\
\left(1+\tau_{x t}\right) \hat{c}_{t}^{-\sigma}\left(1-l_{t}\right)^{\psi(1-\sigma)} /\left(1-\varphi^{\prime}\left(\hat{x}_{t} / \hat{k}_{t}\right)\right) \\
=\hat{\beta} E_{t} \hat{c}_{t+1}^{-\sigma}\left(1-l_{t+1}\right)^{\psi(1-\sigma)}\left[\theta \hat{k}_{t+1}^{\theta-1}\left(z_{t+1} l_{t+1}\right)^{1-\theta}+(1-\delta\right. \\
\left.-\varphi\left(\hat{x}_{t+1} / \hat{k}_{t+1}\right)+\varphi^{\prime}\left(\hat{x}_{t+1} / \hat{k}_{t+1}\right) \hat{x}_{t+1} / \hat{k}_{t+1}\right) \\
\left.\left(1+\tau_{x t+1}\right) /\left(1-\varphi^{\prime}\left(\hat{x}_{t+1} / \hat{k}_{t+1}\right)\right)\right] .
\end{gathered}
$$

Assuming $\varphi(\hat{x} / \hat{k})=\varphi^{\prime}(\hat{x} / \hat{k})=0$, the log-linearization of these equations yields the same results as in the benchmark with the exception of the intertemporal condition:

$$
\begin{aligned}
0 \approx\left(1+\tau_{x}\right)\left\{-\psi(1-\sigma) l /(1-l) \log l_{t}-\sigma \log \hat{c}_{t}+\eta\left(\log \hat{x}_{t}-\log \hat{k}_{t}\right)\right\}+\tau_{x t} \\
-E_{t}\left\{\left(1+\tau_{x}\right)\left\{-\psi(1-\sigma) l /(1-l) \log l_{t+1}-\sigma \log \hat{c}_{t+1}\right\}\right. \\
+\hat{\beta}\left[r(1-\theta)\left(\log l_{t+1}+\log z_{t+1}-\log \hat{k}_{t+1}\right)\right. \\
+\left(1+\tau_{x}\right)\left(1+g_{z}\right)\left(1+g_{n}\right) \eta\left(\log \hat{x}_{t+1}-\log \hat{k}_{t+1}\right) \\
\left.\left.+(1-\delta) \tau_{x t+1}\right]\right\}
\end{aligned}
$$

where $r=\theta \hat{y} / \hat{k}$ and $\eta=\varphi^{\prime \prime}(\hat{x} / \hat{k})(\hat{x} / \hat{k})=a b$.

As before, we guess a solution of the form (A.2.9) and set $\gamma$ 's so that the dynamic residual $(A .2 .17)$ is exactly 0 . We start by deriving $\gamma_{k}$. To do this, we need to write out the coefficients on $\hat{k}_{t+2}, \hat{k}_{t+1}$, and $\hat{k}_{t}$ in the dynamic first-order condition (A.2.17) making use of the $\phi$ 's from the static first-order conditions. For now, we can ignore the expectations operator. We get the following:

$$
\begin{aligned}
0 & =\left[\hat{\beta} r(1-\theta) \phi_{l k^{\prime}}-\left(1+\tau_{x}\right)\left\{\psi(1-\sigma) l /(1-l) \phi_{l k^{\prime}}+\sigma \phi_{c k^{\prime}}-\hat{\beta}\left(1+g_{n}\right)\left(1+g_{z}\right) \eta \phi_{x k^{\prime}}\right\}\right] \hat{k}_{t+2} \\
& +\left[\hat{\beta} r(1-\theta)\left(\phi_{l k}-1\right)-\left(1+\tau_{x}\right)\left\{\psi(1-\sigma) l /(1-l)\left(\phi_{l k}-\phi_{l k^{\prime}}\right)+\sigma\left(\phi_{c k}-\phi_{c k^{\prime}}\right)\right.\right. \\
& \left.\left.-\hat{\beta}\left(1+g_{n}\right)\left(1+g_{z}\right) \eta\left(\phi_{x k}-1\right)+\eta \phi_{x k^{\prime}}\right\}\right] \hat{k}_{t+1} \\
& +\left[\left(1+\tau_{x}\right)\left\{\psi(1-\sigma) l /(1-l) \phi_{l k}+\sigma \phi_{c k}-\eta\left(\phi_{x k}-1\right)\right\}\right] \hat{k}_{t} \\
& + \text { all other terms }
\end{aligned}
$$


or, more succinctly, rewrite $(A .2 .18)$ as $\left(a+b L+c L^{2}\right) \hat{k}_{t+2}=$ other terms, where $\gamma_{k}$ is the root of the quadratic inside the unit circle. Note that $\gamma_{k}$ does not depend on the other unknown $\gamma$ 's.

Given $\gamma_{k}$, we can solve a linear system for the other $\gamma^{\prime}$ s. At this point, we do not ignore the expectations operator:

$$
\begin{aligned}
& 0 \approx\left(1+\tau_{x}\right)\left\{-\psi(1-\sigma) l /(1-l)\left[\left(\phi_{l z}+\phi_{l k^{\prime}} \gamma_{z}\right) \log z_{t}+\left(\phi_{l l}+\phi_{l k^{\prime}} \gamma_{l}\right) \tau_{l t}\right.\right. \\
& \left.+\phi_{l k^{\prime}} \gamma_{x} \tau_{x t}+\left(\phi_{l g}+\phi_{l k^{\prime}} \gamma_{g}\right) \log \hat{g}_{t}\right] \\
& -\sigma\left[\left(\phi_{c z}+\phi_{c k^{\prime}} \gamma_{z}\right) \log z_{t}+\left(\phi_{c l}+\phi_{c k^{\prime}} \gamma_{l}\right) \tau_{l t}\right. \\
& \left.+\phi_{c k^{\prime}} \gamma_{x} \tau_{x t}+\left(\phi_{c g}+\phi_{c k^{\prime}} \gamma_{l}\right) \log \hat{g}_{t}\right] \\
& \left.+\eta \phi_{x k^{\prime}}\left[\gamma_{z} \log z_{t}+\gamma_{l} \tau_{l t}+\gamma_{x} \tau_{x t}+\gamma_{g} \log \hat{g}_{t}\right]\right\}+\tau_{x t} \\
& -E_{t}\left(1+\tau_{x}\right)\left\{-\psi(1-\sigma) l /(1-l)\left[\left(\phi_{l k}+\phi_{l k^{\prime}} \gamma_{k}\right)\left(\gamma_{z} \log z_{t}+\gamma_{l} \tau_{l t}+\gamma_{x} \tau_{x t}+\gamma_{g} \log \hat{g}_{t}\right)\right.\right. \\
& +\left(\phi_{l z}+\phi_{l k^{\prime}} \gamma_{z}\right) \log z_{t+1} \\
& +\left(\phi_{l l}+\phi_{l k^{\prime}} \gamma_{l}\right) \tau_{l t+1} \\
& +\phi_{l k^{\prime}} \gamma_{x} \tau_{x t+1} \\
& \left.+\left(\phi_{l g}+\phi_{l k^{\prime}} \gamma_{g}\right) \log \hat{g}_{t+1}\right] \\
& -\sigma\left[\left(\phi_{c k}+\phi_{c k^{\prime}} \gamma_{k}\right)\left(\gamma_{z} \log z_{t}+\gamma_{l} \tau_{l t}+\gamma_{x} \tau_{x t}+\gamma_{g} \log \hat{g}_{t}\right)\right. \\
& +\left(\phi_{c z}+\phi_{c k^{\prime}} \gamma_{z}\right) \log z_{t+1} \\
& +\left(\phi_{c l}+\phi_{c k^{\prime}} \gamma_{l}\right) \tau_{l t+1} \\
& +\phi_{c k^{\prime}} \gamma_{x} \tau_{x t+1} \\
& \left.\left.+\left(\phi_{c g}+\phi_{c k^{\prime}} \gamma_{g}\right) \log \hat{g}_{t+1}\right]\right\}
\end{aligned}
$$




$$
\begin{aligned}
& -\hat{\beta} r(1-\theta) E_{t}\left[\left(\phi_{l k}+\phi_{l k^{\prime}} \gamma_{k}-1\right)\left(\gamma_{z} \log z_{t}+\gamma_{l} \tau_{l t}+\gamma_{x} \tau_{x t}+\gamma_{g} \log \hat{g}_{t}\right)\right. \\
& +\left(\phi_{l z}+\phi_{l k^{\prime}} \gamma_{z}+1\right) \log z_{t+1} \\
& +\left(\phi_{l l}+\phi_{l k^{\prime}} \gamma_{l}\right) \tau_{l t+1} \\
& +\phi_{l k^{\prime}} \gamma_{x} \tau_{x t+1} \\
& \left.+\left(\phi_{l g}+\phi_{l k^{\prime}} \gamma_{g}\right) \log \hat{g}_{t+1}\right] \\
& -\hat{\beta}\left(1+\tau_{x}\right)\left(1+g_{n}\right)\left(1+g_{z}\right) \eta E_{t}\left[\left(\phi_{x k}+\phi_{x k^{\prime}} \gamma_{k}-1\right)\left(\gamma_{z} \log z_{t}+\gamma_{l} \tau_{l t}+\gamma_{x} \tau_{x t}+\gamma_{g} \log \hat{g}_{t}\right)\right. \\
& +\phi_{x k^{\prime}}\left(\gamma_{z} \log z_{t+1}+\gamma_{l} \tau_{l t+1}+\gamma_{x} \tau_{x t+1}+\gamma_{g} \log \hat{g}_{t+1}\right) \\
& -\hat{\beta}(1-\delta) E_{t} \tau_{x t+1}, \quad
\end{aligned}
$$

which reduces to a system like $(A .2 .11)$.

\section{A.2.4. An Alternative Investment Wedge}

In a comment on our paper, Christiano and Davis (2006) note that our findings may be sensitive to the particular choice of the intertemporal wedge. In theory the choice of the intertemporal wedge should not matter in the sense that it just implies a (slightly) different map between the detailed economy and the prototype economy. However, in practice, stochastic processes for the wedges are estimated, and therefore we want to make sure that our substantive findings are not affected by the choice. Here we describe Christiano and Davis' (2006) alternative investment wedge, and later we demonstrate that our findings are not sensitive to this alternative. ${ }^{2}$

Christiano and Davis (2006) assume that the intertemporal wedge is $\tau_{k}$ and that the dynamic first-order condition (with adjustment costs) is given by

$$
\begin{aligned}
& U_{1}\left(\hat{c}_{t}, 1-l_{t}\right) /\left(1-\varphi^{\prime}\left(\hat{x}_{t} / \hat{k}_{t}\right)\right) \\
& \quad=\hat{\beta} E_{t}\left\{U _ { 1 } ( \hat { c } _ { t + 1 } , 1 - l _ { t + 1 } ) ( 1 - \tau _ { k t + 1 } ) \left[r_{t+1}+\frac{1}{1-\varphi^{\prime}\left(\hat{x}_{t+1} / \hat{k}_{t+1}\right)}\right.\right.
\end{aligned}
$$

2 We also explain later why the methodology used by Christiano and Davis (2006) — which is not the methodology used in the final version of our paper - can lead to a different conclusion. 


$$
\left.\left.\cdot\left\{1-\delta-\varphi\left(\frac{\hat{x}_{t+1}}{\hat{k}_{t+1}}\right)+\varphi^{\prime}\left(\frac{\hat{x}_{t+1}}{\hat{k}_{t+1}}\right) \frac{\hat{x}_{t+1}}{\hat{k}_{t+1}}\right\}\right]\right\} .
$$

Here, $\tau_{k}$ resembles a tax on the gross return to capital. In Appendix C, we compare our predictions based on the $\tau_{k}$ wedge with those based on the $\tau_{x}$ wedge. $^{3}$

\section{A.3. MLE Estimation}

We now describe the general method we use to estimate the processes governing the four exogenous variables in $s_{t}$ with the data described above.

\section{A.3.1. State-Space Form}

$$
\begin{aligned}
X_{t+1} & =A X_{t}+B \epsilon_{t+1} \\
Y_{t} & =C X_{t}+\omega_{t} \\
\omega_{t} & =D \omega_{t-1}+\eta_{t},
\end{aligned}
$$

where $X_{t}=\left[\log \hat{k}_{t}, \log z_{t}, \tau_{l t}, \tau_{x t}, \log \hat{g}_{t}, 1\right]^{\prime}, Y_{t}=\left[\log \hat{y}_{t}, \log \hat{x}_{t}, \log l_{t}, \log \hat{g}_{t}\right]$, and

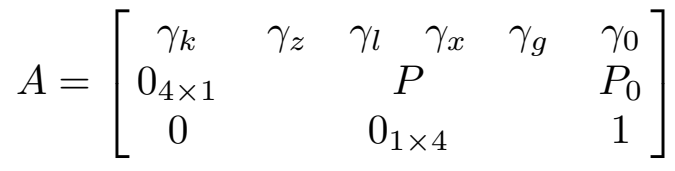

$$
\begin{aligned}
& B=\left[\begin{array}{c}
0_{1 \times 4} \\
Q \\
0
\end{array}\right] \\
& C=\left[\begin{array}{cccccc}
\phi_{y k} & \phi_{y z} & \phi_{y l} & 0 & \phi_{y g} & \phi_{y 0} \\
\phi_{x k} & 0 & 0 & 0 & 0 & \phi_{x 0} \\
\phi_{l k} & \phi_{l z} & \phi_{l l} & 0 & \phi_{l g} & \phi_{l 0} \\
0 & 0 & 0 & 0 & 1 & 0
\end{array}\right]+\left[\begin{array}{c}
\phi_{y k^{\prime}} \\
\phi_{x k^{\prime}} \\
\phi_{l k^{\prime}} \\
0
\end{array}\right]\left[\begin{array}{llllll}
\gamma_{k} & \gamma_{z} & \gamma_{l} & \gamma_{x} & \gamma_{g} & 0
\end{array}\right]
\end{aligned}
$$

and elements of $D$ are the parameters governing serial correlation of the measurement error. Assume that $E \eta_{t} \eta_{t}^{\prime}=R, E \epsilon_{t} \eta_{s}^{\prime}=0$ for all periods $t$ and $s$. Define $\bar{Y}_{t} \equiv$

3 We report results only for the postwar period applying a log-linear approximation method, so we have dropped the penalty functions needed for our nonlinear solution method. 
$Y_{t+1}-D Y_{t}$. Then we can rewrite the system as

$$
\begin{aligned}
X_{t+1} & =A X_{t}+B \epsilon_{t+1} \\
\bar{Y}_{t} & =\bar{C} X_{t}+C B \epsilon_{t+1}+\eta_{t+1} .
\end{aligned}
$$

\section{A.3.2. Log-Likelihood Function}

$$
L(\Theta)=\sum_{t=0}^{T-1}\left\{\log \left|\Omega_{t}\right|+\operatorname{trace}\left(\Omega_{t}^{-1} u_{t} u_{t}^{\prime}\right)-\log \left|\partial f\left(Z_{t}, \Theta\right) / \partial Z_{t}\right|\right\},
$$

where the parameters to be estimated are stacked in vector $\Theta$, the innovation vector is $u_{t}$, and its covariance is $\Omega_{t}$. The last term in (A.3.1) is nonzero if the elements of $Y$ are not the raw series but depend on the raw series $Z$ plus the parameter vector. For example, if we estimate $g_{z}$ and use per-capita values as our raw data, then $Z$ is per-capita data and $Y$ is detrended per-capita data.

The innovation vector $u_{t}$ and its covariance $\Omega_{t}$ are defined as follows:

$$
\begin{aligned}
u_{t} & =\bar{Y}_{t}-\hat{E}\left[\bar{Y}_{t} \mid \bar{Y}_{t-1}, \bar{Y}_{t-2}, \ldots, \bar{Y}_{0}, \hat{X}_{0}\right] \\
& =Y_{t+1}-\hat{E}\left[Y_{t+1} \mid Y_{t}, Y_{t-1}, \ldots, Y_{0}, \hat{X}_{0}\right] \\
& =Y_{t+1}-D Y_{t}-\bar{C} \hat{X}_{t} \\
\Omega_{t} & =E u_{t} u_{t}^{\prime}=\bar{C} \Sigma_{t} \bar{C}^{\prime}+R+C B B^{\prime} C^{\prime},
\end{aligned}
$$

which in turn depends on the predicted state $\hat{X}_{t}$ :

$$
\hat{X}_{t}=\hat{E}\left[X_{t} \mid Y_{t}, Y_{t}, \ldots, Y_{0}, \hat{X}_{0}\right] .
$$

The predicted state evolves according to

$$
\hat{X}_{t+1}=A \hat{X}_{t}+K_{t} u_{t}
$$

where $K_{t}$ is the Kalman gain,

$$
\begin{aligned}
K_{t} & =\left(B B^{\prime} C^{\prime}+A \Sigma_{t} \bar{C}^{\prime}\right) \Omega_{t}^{-1} \\
\Sigma_{t+1} & =A \Sigma_{t} A^{\prime}+B B^{\prime}-\left(B B^{\prime} C^{\prime}+A \Sigma_{t} \bar{C}^{\prime}\right) \Omega_{t}^{-1}\left(\bar{C} \Sigma_{t} A^{\prime}+C B B^{\prime}\right)
\end{aligned}
$$


with state covariance $\Sigma_{t}$.

For the results in the paper, we fixed parameters of preferences, production, and growth and estimated the processes for the wedges. The parameters that were fixed were $\psi=2.24, \sigma=1, \beta=0.9722, \theta=0.35, \delta=0.0464, g_{n}=1.5 \%$, and $g_{z}=1.6 \%$. We also set the measurement errors equal to zero $\left(D=R=0_{4 \times 4}\right)$ in all periods and all numerical experiments. ${ }^{4}$ The parameters that were estimated were elements of $P_{0}, P$, and $Q$.

The parameter choices were based on time series during the pre-World War II period. There have been some modest changes in growth rates, depreciation rates, and capital shares in the post-World War II period. Since we separately estimate the means of the wedges in the pre- and postwar periods, our main results are not affected. One advantage of keeping the utility and technology parameters fixed is that we can back out wedges for the entire century. (These are shown later in Figures A1 and A2.)

\section{A.4. Decomposing Macro Aggregates}

Here, we describe the details of our implementing our accounting procedure for the Great Depression and postwar periods. They differ slightly because the equilibria are computed differently in the two periods: we use a nonlinear computational routine for the Great Depression and a log-linear computational routine for the postwar period.

\section{A.4.1. Great Depression Period}

Because shocks are large during the Great Depression period, we need to compute equilibria nonlinearly. We use a large (i.e., 459 state) Markov chain to approximate the process for the shocks to avoid having to compute a five-dimensional continuous-state model.

Specifically, using estimates for the matrices underlying the stochastic process on

\footnotetext{
4 This choice makes no difference for our results.
} 
wedges, namely $P_{0}, P$, and $Q$, we construct a Markov transition matrix using a methodology that is similar to-but not the same as-Tauchen (1986). The difference is that Tauchen rewrites the problem to have a diagonal covariance matrix on the disturbances and we assume a dense covariance matrix. We do this because it allows us more flexibility in choosing the grid over our state variables.

Given a Markov chain, we want to find the realization of the wedges, one consistent with the estimated process, that implies exact agreement between model simulations and observations. For $\log z_{t}$, we use the specified production function above and observations on output, labor input, and the capital stock (accumulated via perpetual inventory with the observed investment series). For $\tau_{l t}$, we use the static first-order condition $(A .2 .2)$ along with series for capital, labor, consumption, and $z_{t}$. For $\log g_{t}$, we have a direct measure.

We cannot infer a realization for $\tau_{x t}$ directly from static first-order conditions. Instead, we find the realization of $\tau_{x t}$ that gives an exact match of the model simulation and U.S. observations over a specified period (e.g., the Great Depression or the 1982 recession). We start by making a guess for the series $\tau_{x t}$ for the period in which we are interested. For example, when we examine the period of the Great Depression, we make a guess about the realization of $\tau_{x t}$ from 1929 through 1939. A good starting point for this guess can be derived from the log-linear decision rule on investment, which is a function of the capital stock and the other wedges. This amounts to solving one equation in one unknown $\left(\tau_{x}\right)$.

With a guess for the realization of $\tau_{x t}$ and realizations for all of the other wedges, we simulate the model as follows. We take our Markov chain for the wedges (found using the variation on Tauchen's method) and append it by $N$ states, where $N$ is the number of years in the period we want to study. Suppose that the original chain had $M$ states. The appended chain has $M+N$. In the case of the Great Depression, for example, we have $N=11$, which is the length of the period 1929-1939. The first of the appended states 
corresponds to the first year; the first year is 1929 in the case of the Great Depression. We have values for $z_{1929}, \tau_{l 1929}, \hat{g}_{1929}$. We have a guess for $\tau_{x 1929}$. For each of the $N$ additional states, we use our version of Tauchen's method to compute the transition problems from this state to the $M$ original states. Because we want a sequence for $\tau_{x t}$ during the Great Depression that generates an exact match between data and model for all wedges on, we have to iterate (reguessing the sequence and updating the Markov chain transition) until there is an exact match. The derived wedges are shown in Figure 1 of the paper.

Once we have this realization for all of the wedges - one that can exactly generate the data - we turn off one or more of the wedges by setting them at their initial levels (say, 1929 in the case of the Depression). For example, to see how important $\log z$ is in the Great Depression, we can set $\tau_{x}, \tau_{l}$, and $\hat{g}$ (in all states) equal to their 1929 levels. We hold fixed the underlying stochastic process. This means that we hold the Markov chain transition probabilities fixed for each new simulation. The results of the one-wedge-alone or one-wedge-off experiments are shown in Figures 2-4.

\section{A.4.2. Postwar Period}

In the postwar period, there is no need to approximate the stochastic process for the wedges using a Markov Chain because the shocks are much smaller. In this case, a loglinear approximation of the five-dimensional, continuous state model works well.

As in the Great Depression, we fix the stochastic process $s^{t}$ when considering the marginal effect of one wedge. To accomplish that, we set each of the wedges equal to the sum of a constant times an indicator function plus the corresponding state in $s$ times one minus the indicator function; for example,

$$
\tau_{l t}=\chi \bar{\tau}_{l}+(1-\chi) s_{2 t},
$$

where $s_{2 t}$ is the second element of the state vector $s_{t}$ and $\chi$ is equal to 0 or 1 depending 
on whether variations in the wedge $\tau_{l t}$ are being analyzed or not, respectively. ${ }^{5}$

\section{A.5. A Model with Capacity Utilization}

In the cases where we allow for capacity utilization (Figures 9-12 in the paper), we use the following functional form for technology:

$$
F(k, z l)=k^{\theta} z l
$$

and redo the steps outlined above.

In the log-linearized solution, we have the following new first-order conditions:

$$
\begin{aligned}
& \hat{c}_{t}+\hat{g}_{t}+\left(1+g_{n}\right)\left(1+g_{z}\right) \hat{k}_{t+1}-(1-\delta) \hat{k}_{t}=\hat{k}_{t}^{\theta} z_{t} l_{t} \\
& \psi \hat{c}_{t}=\left(1-\tau_{l t}\right) \hat{k}_{t}^{\theta} z_{t}\left(1-l_{t}\right) \\
& \left(1+\tau_{x t}\right) \hat{c}_{t}^{-\sigma}\left(1-l_{t}\right)^{\psi(1-\sigma)} \\
& =\hat{\beta} E_{t} \hat{c}_{t+1}^{-\sigma}\left(1-l_{t+1}\right)^{\psi(1-\sigma)} \\
& {\left[\theta \hat{k}_{t+1}^{\theta-1} z_{t+1} l_{t+1}+(1-\delta)\left(1+\tau_{x t+1}\right)\right] .}
\end{aligned}
$$

The new steady state in this case is $\hat{k}$ and $l$ that solve

$$
\begin{aligned}
& \psi\left[\hat{k}^{\theta} z l-\left(1+g_{n}\right)\left(1+g_{z}\right) \hat{k}+(1-\delta) \hat{k}-\hat{g}\right]=\left(1-\tau_{l}\right) \hat{k}^{\theta} z(1-l) \\
& \left(1+\tau_{x}\right)=\hat{\beta}\left[\theta \hat{k}^{\theta-1} z l+(1-\delta)\left(1+\tau_{x}\right)\right] .
\end{aligned}
$$

Solving this system of equations is like solving a problem of the form $\xi_{1} \hat{k}-\xi_{2}=\xi_{3} \hat{k}^{\theta}$,

5 In earlier versions of the paper we set $\tau_{l t}=s_{2 t}$ and set it equal to a constant when its effects were not being analyzed. The quantitative impact was very small for our prototype model with a $\tau_{x}$ investment wedge. They are not small for Christiano and Davis (2006), who prefer to use a $\tau_{k}$ wedge and to set adjustment costs very high. In the published version of our paper, we described our current procedure which is consistent with our propositions that separate the direct effects of fluctuations in the wedges with indirect effects due to forecasting fluctuations in other wedges. Later, we show that if Christiano and Davis (2006) were to apply the procedure that is consistent with our propositions, their predictions would line up almost exactly with ours. 
where

$$
\begin{aligned}
& \xi_{1}=\left(\psi+1-\tau_{l}\right) \xi-\psi\left[\left(1+g_{n}\right)\left(1+g_{z}\right)-1+\delta\right] \\
& \xi_{2}=\psi \hat{g} \\
& \xi_{3}=\left(1-\tau_{l}\right) z \\
& \xi=\left(1+\tau_{x}\right)[1-\hat{\beta}(1-\delta)] /(\hat{\beta} \theta) .
\end{aligned}
$$

We compute $\hat{k}$ with a simple Newton algorithm starting with $\hat{k}=\left(\xi_{3} / \xi_{1}\right)^{1 /(1-\theta)}$, which is the exact solution if $\hat{g}=0$. The solution for $\hat{k}$ in the $\hat{g}>0$ case will be higher. Once we have $\hat{k}$, we have $l=\xi \hat{k}^{1-\theta} / z$.

The new log-linearized first-order conditions (ignoring constants) imply the following for detrended consumption:

$$
\begin{aligned}
\hat{c}_{t} \approx & \hat{c} \log \hat{c}_{t} \\
\approx & \hat{k}^{\theta} z l\left[\theta \log \hat{k}_{t}+\log z_{t}+\log l_{t}\right] \\
& -\left(1+g_{z}\right)\left(1+g_{n}\right) \hat{k} \log \hat{k}_{t+1}+(1-\delta) \hat{k} \log \hat{k}_{t}-\hat{g} \log \hat{g}_{t} .
\end{aligned}
$$

As before, the labor input is derived from the static first-order condition $(A .2 .2)$

$$
\begin{aligned}
& 0 \approx \psi\left\{\hat{k}^{\theta} z l\left[\theta \log \hat{k}_{t}+\log z_{t}+\log l_{t}\right]\right. \\
& \left.-\left(1+g_{z}\right)\left(1+g_{n}\right) \hat{k} \log \hat{k}_{t+1}+(1-\delta) \hat{k} \log \hat{k}_{t}-\hat{g} \log \hat{g}_{t}\right\} \\
& +\left(1-\tau_{l}\right) \hat{k}^{\theta} z(1-l)\left\{1 /\left(1-\tau_{l}\right) \tau_{l t}\right. \\
& \left.-\theta \log \hat{k}_{t}-\log z_{t}+l /(1-l) \log l_{t}\right\}
\end{aligned}
$$

which we write succinctly as

$$
\log l_{t}=\phi_{l k} \log \hat{k}_{t}+\phi_{l z} \log z_{t}+\phi_{l l} \tau_{l t}+\phi_{l g} \log \hat{g}_{t}+\phi_{l k^{\prime}} \log \hat{k}_{t+1}
$$

Using this equation for $\log l$, we use the other static first-order conditions to derive output, 
investment, and consumption as follows:

$$
\begin{aligned}
\log \hat{y}_{t}= & \phi_{y k} \log \hat{k}_{t}+\phi_{y z} \log z_{t}+\phi_{y l} \tau_{l t}+\phi_{y k^{\prime}} \log \hat{k}_{t+1} \\
= & \left(\theta+\phi_{l k}\right) \log \hat{k}_{t}+\left(1+\phi_{l z}\right) \log z_{t} \\
& +\phi_{l l} \tau_{l t}+\phi_{l k^{\prime}} \log \hat{k}_{t+1} \\
\log \hat{x}_{t}= & \left(1+g_{z}\right)\left(1+g_{n}\right) \hat{k} / \hat{x} \log \hat{k}_{t+1}-(1-\delta) \hat{k} / \hat{x} \log \hat{k}_{t} \\
\log \hat{c}_{t}= & \phi_{c k} \log \hat{k}_{t}+\phi_{c z} \log z_{t}+\phi_{c l} \tau_{l t}+\phi_{c g} \log \hat{g}_{t}+\phi_{c k^{\prime}} \log \hat{k}_{t+1} \\
= & {\left[\hat{y} \log y_{t}-\hat{x} \log x_{t}-\hat{g} \log \hat{g}_{t}\right] / \hat{c} }
\end{aligned}
$$

The new dynamic first-order condition implies the following for capital:

$$
\begin{aligned}
& 0 \approx\left(1+\tau_{x}\right) \hat{c}^{-\sigma}(1-l)^{\psi(1-\sigma)}\left\{-\psi(1-\sigma) l /(1-l) \log l_{t}-\sigma \log \hat{c}_{t}\right\} \\
& +\hat{c}^{-\sigma}(1-l)^{\psi(1-\sigma)} \tau_{x t} \\
& -\hat{\beta} E_{t}\left\{\left[\theta \hat{k}^{\theta-1} z l+(1-\delta)\left(1+\tau_{x}\right)\right]\right. \\
& \cdot\left[\hat{c}^{-\sigma}(1-l)^{\psi(1-\sigma)}\left\{-\psi(1-\sigma) l /(1-l) \log l_{t+1}-\sigma \log \hat{c}_{t+1}\right\}\right] \\
& +\hat{c}^{-\sigma}(1-l)^{\psi(1-\sigma)}\left[\theta \hat{k}^{\theta-1} z l\right. \\
& \left.\left.\quad \cdot\left(\log l_{t+1}+\log z_{t+1}-(1-\theta) \log \hat{k}_{t+1}\right)+(1-\delta) \tau_{x t+1}\right]\right\}
\end{aligned}
$$

which simplifies to

$$
\begin{aligned}
0 \approx(1+ & \left.\tau_{x}\right)\left\{-\psi(1-\sigma) l /(1-l) \log l_{t}-\sigma \log \hat{c}_{t}\right\}+\tau_{x t} \\
- & E_{t}\left\{\left(1+\tau_{x}\right)\left\{-\psi(1-\sigma) l /(1-l) \log l_{t+1}-\sigma \log \hat{c}_{t+1}\right\}\right. \\
& \left.+\hat{\beta}\left[r\left(\log l_{t+1}+\log z_{t+1}-(1-\theta) \log \hat{k}_{t+1}\right)+(1-\delta) \tau_{x t+1}\right]\right\}
\end{aligned}
$$

where $r=\theta \hat{y} / \hat{k}$.

The form of the solution is given by $(A .2 .9)$. To compute $\gamma_{k}$, we need to write out the coefficients on $\hat{k}_{t+2}, \hat{k}_{t+1}$, and $\hat{k}_{t}$ in the dynamic first-order condition making use of the $\phi$ 's from the static first-order condition. For now, we can ignore the expectations operator. 
We get the following:

$$
\begin{gathered}
0 \approx-\left(1+\tau_{x}\right)\left\{-\psi(1-\sigma) l /(1-l)\left[\phi_{l k} \log \hat{k}_{t}+\phi_{l k^{\prime}} \log \hat{k}_{t+1}\right]\right. \\
\left.-\sigma\left[\phi_{c k} \log \hat{k}_{t}+\phi_{c k^{\prime}} \log \hat{k}_{t+1}\right]\right\} \\
+E_{t}\left(1+\tau_{x}\right)\left\{-\psi(1-\sigma) l /(1-l)\left[\phi_{l k} \log \hat{k}_{t+1}+\phi_{l k^{\prime}} \log \hat{k}_{t+2}\right]\right. \\
\left.-\sigma\left[\phi_{c k} \log \hat{k}_{t+1}+\phi_{c k^{\prime}} \log \hat{k}_{t+2}\right]\right\} \\
+\hat{\beta} E_{t}\left[r\left(\phi_{l k} \log \hat{k}_{t+1}+\phi_{l k^{\prime}} \hat{k}_{t+2}-(1-\theta) \log \hat{k}_{t+1}\right)\right]
\end{gathered}
$$

which simplifies to

$$
\begin{aligned}
& 0=\left[\hat{\beta} r \phi_{l k^{\prime}}-\left(1+\tau_{x}\right) \psi(1-\sigma) l /(1-l) \phi_{l k^{\prime}}-\left(1+\tau_{x}\right) \sigma \phi_{c k^{\prime}}\right] \hat{k}_{t+2} \\
& +\left[\hat{\beta} r\left(\phi_{l k}-1+\theta\right)-\left(1+\tau_{x}\right) \psi(1-\sigma) l /(1-l) \phi_{l k}-\left(1+\tau_{x}\right) \sigma \phi_{c k}\right. \\
& \left.+\left(1+\tau_{x}\right) \psi(1-\sigma) l /(1-l) \phi_{l k^{\prime}}+\left(1+\tau_{x}\right) \sigma \phi_{c k^{\prime}}\right] \hat{k}_{t+1} \\
& -\left[-\left(1+\tau_{x}\right) \psi(1-\sigma) l /(1-l) \phi_{l k}-\left(1+\tau_{x}\right) \sigma \phi_{c k}\right] \hat{k}_{t} \\
& + \text { all other terms. }
\end{aligned}
$$

Given $\gamma_{k}$, we solve a linear system for the other $\gamma^{\prime}$ 's. In particular, we have

$$
\begin{gathered}
0 \approx\left(1+\tau_{x}\right)\left\{-\psi(1-\sigma) l /(1-l)\left[\left(\phi_{l z}+\phi_{l k^{\prime}} \gamma_{z}\right) \log z_{t}\right.\right. \\
+\left(\phi_{l l}+\phi_{l k^{\prime}} \gamma_{l}\right) \tau_{l t} \\
+\phi_{l k^{\prime}} \gamma_{x} \tau_{x t}+\left(\phi_{l g}+\phi_{l k^{\prime}} \gamma_{g}\right) \log \hat{g}_{t} \\
-\sigma\left[\left(\phi_{c z}+\phi_{c k^{\prime}} \gamma_{z}\right) \log z_{t}+\left(\phi_{c l}+\phi_{c k^{\prime}} \gamma_{l}\right) \tau_{l t}\right. \\
\left.\left.+\phi_{c k^{\prime}} \gamma_{x} \tau_{x t}+\left(\phi_{c g}+\phi_{c k^{\prime}} \gamma_{l}\right) \log \hat{g}_{t}\right]\right\}+\tau_{x t} \\
-E_{t}\left(1+\tau_{x}\right)\left\{-\psi(1-\sigma) l /(1-l)\left[\left(\phi_{l k}+\phi_{l k^{\prime}} \gamma_{k}\right)\left(\gamma_{z} \log z_{t}+\gamma_{l} \tau_{l t}+\gamma_{x} \tau_{x t}\right)\right.\right. \\
+\left(\phi_{l z}+\phi_{l k^{\prime}} \gamma_{z}\right) \log z_{t+1} \\
+\left(\phi_{l l}+\phi_{l k^{\prime}} \gamma_{l}\right) \tau_{l t+1} \\
+\phi_{l k^{\prime}} \gamma_{x} \tau_{x t+1} \\
\left.+\left(\phi_{l g}+\phi_{l k^{\prime}} \gamma_{g}\right) \log \hat{g}_{t+1}\right]
\end{gathered}
$$




$$
\begin{gathered}
-\sigma\left[\left(\phi_{c k}+\phi_{c k^{\prime}} \gamma_{k}\right)\left(\gamma_{z} \log z_{t}+\gamma_{l} \tau_{l t}+\gamma_{x} \tau_{x t}+\gamma_{g} \log \hat{g}_{t}\right)\right. \\
+\left(\phi_{c z}+\phi_{c k^{\prime}} \gamma_{z}\right) \log z_{t+1} \\
+\left(\phi_{c l}+\phi_{c k^{\prime}} \gamma_{l}\right) \tau_{l t+1} \\
+\phi_{c k^{\prime}} \gamma_{x} \tau_{x t+1} \\
\left.\left.+\left(\phi_{c g}+\phi_{c k^{\prime}} \gamma_{g}\right) \log \hat{g}_{t+1}\right]\right\} \\
-\hat{\beta} r E_{t}\left[\left(\phi_{l k}+\phi_{l k^{\prime}} \gamma_{k}-1+\theta\right)\left(\gamma_{z} \log z_{t}+\gamma_{l} \tau_{l t}+\gamma_{x} \tau_{x t}\right)\right. \\
\quad+\left(\phi_{l z}+\phi_{l k^{\prime}} \gamma_{z}+1\right) \log z_{t+1} \\
\quad+\left(\phi_{l l}+\phi_{l k^{\prime}} \gamma_{l}\right) \tau_{l t+1} \\
+\phi_{l k^{\prime}} \gamma_{x} \tau_{x t+1} \\
\left.+\left(\phi_{l g}+\phi_{l k^{\prime}} \gamma_{g}\right) \log \hat{g}_{t+1}\right] \\
-\hat{\beta}(1-\delta) E_{t} \tau_{x t+1},
\end{gathered}
$$

and after this, the procedure is the same as for the benchmark model. 


\section{Appendix B. Data and Sources}

In this Appendix, we describe our data and their sources. Early drafts of the paper reported results for the annual data described in Section 1. We subsequently redid the exercises for the postwar period using quarterly data (which can be updated by future users of the codes). The Bureau of Economic Analysis (BEA) has done comprehensive revisions of the national accounts and reenumerated many of the standard national income and product account (NIPA) tables. Thus, the NIPA tables with annual data do not correspond to the current naming scheme of the BEA. We do have all of the original tables that we used in this project, along with documentation.

\section{B.1. U.S. Historical Annual Data Measures and Sources}

Here, we provide a list of the variables in the model and their data analogs. Because we work with data going back to 1900, the main output series for the annual data is gross national product.

\section{B.1.1. Measures}

- Per-capita output $(y)$

GNP

- Sales tax

- Military compensation

+ Services from consumer durables (with return $=4 \%$ )

+ Depreciation from consumer durables

- Per-capita investment $(x)$ 
Gross private fixed investment

+ Private inventories

+ Government gross investment

+ Net factor payments (GNP-GDP)

+ Personal consumption expenditures on durables

- Sales tax $\times$ share of durables in PCE

All deflated by the GNP deflator and Population over 16

- Per-capita government $(g)$

Government consumption

- Military compensation

+ Military equipment

$-1 / 2$ Military facilities

+ Net exports of goods and services

All deflated by the GNP deflator and Population over 16

- Per-capita labor input $(l)$

(Civilian annual manhours / Population over 16) / (50 weeks $\times 100$ hours $)$

\section{B.1.2. Sources}

The specific sources of the data listed above are as follows:

- National accounts, pre-1929

Kendrick (1961), Table A-IIb (all mil. \$)

Total consumption expenditures

New construction and equipment

Change in business inventories 
Net foreign investment

Government purchases of goods and services

GNP (commerce concept)

Kendrick (1961), Table A-IIa (in mil. 1929 \$)

GNP

Change in business inventories

○ National accounts, post-1929

www.bea.gov, old NIPA Table 1.1 (in bil. \$)

Gross domestic product (GDP)

Personal consumption expenditures (PCE)

PCE durable goods

PCE nondurable goods

PCE services

Gross private domestic investment (GPDI)

GPDI fixed investment

GPDI change in private inventories

Net exports of goods and services

Government consumption expenditures and gross investment

www.bea.gov, old NIPA Table 1.9 (in bil. \$)

Gross national product (GNP)

www.bea.gov, old NIPA Table 3.9 (in bil. \$)

Government consumption expenditures

www.bea.gov, old NIPA Table 5.1 (in bil. \$)

Gross government investment 
www.bea.gov, old NIPA Table 3.7 (in bil. \$)

National defense gross investment in structures

National defense gross investment in equipment and software

www.bea.gov, old NIPA Table 3.5 (in bil. \$)

Federal excise taxes

State and local sales taxes

State and local other taxes

www.bea.gov, old NIPA Table 6.2 (in mil. \$)

Compensation of military employees

www.bea.gov, old NIPA Table 6.3 (in mil. \$)

Wage and salary accruals for military

- Fixed assets and investments

www.bea.gov, FA Table 1.1 (in mil. \$ year-end)

Current-cost net stock of fixed assets, private

Current-cost net stock of consumer durable goods

www.bea.gov, FA Table 7.1 (in mil. $\$$ year-end)

Current-cost net stock of fixed assets, government

Current-cost net stock of fixed assets, government national defense, equipment and software

Current-cost net stock of fixed assets, government national defense, military facilities

www.bea.gov, FA Table 1.5 (in mil. \$)

Historical-cost investment in fixed assets, private

Historical-cost investment in consumer durable goods 
www.bea.gov, FA Table 7.5 (in mil. \$)

Historical-cost investment in fixed assets, government

Historical-cost investment in fixed assets, government national defense, equipment and software

Historical-cost investment in fixed assets, government national defense, military facilities

- Civilian manhours, pre-1947

Kendrick (1961), Table A-X (millions)

○ Civilian manhours, post-1947

www.bea.gov, old NIPA Table 6.5 (thousands)

Full-time equivalent employees

Full-time equivalent employees, military

www.bea.gov, old NIPA Table 6.8 (thousands)

Persons engaged in production

Persons engaged in production, military

www.bea.gov, old NIPA Table 6.9 (mil. of hours)

Hours worked by full-time and part-time employees

○ Population over 16

Historical Statistics, Series A6-8

Economic Report of the President (2001), Table B-34. 


\section{B.1.3. Appendix Figures}

Figures A1 and A2 are the efficiency and tax wedges, respectively, over the entire twentieth century.

\section{B.2. U.S. Postwar Quarterly Data Measures and Sources}

For the quarterly series, we have total hours data only after 1959:1. Since there are no world wars in our postwar sample, we do not adjust for military compensation. The main output series that we use for the postwar series is gross domestic product.

\section{B.2.1. Measures}

- Per-capita output $(y)$

Real GDP

- Sales tax deflated by the personal consumption expenditures (PCE) deflator

+ Services from consumer durables (with return $=4 \%$ ) deflated by the PCE durable deflator

+ Depreciation from consumer durables deflated by the PCE durable deflator All divided by non-institutional population $16-64$

- Per-capita investment $(x)$

Real gross private domestic investment (fixed plus inventories)

+ Real government gross investment

+ Real personal consumption expenditures on durables

- Sales tax deflated by PCE deflator $\times$ share of durables in PCE

All divided by non-institutional population $16-64$

- Per-capita government $(g)$ 
Real government consumption

+ Real net exports of goods and services

All divided by non-institutional population 16-64

- Per-capita labor input $(l)$

Total hours from the current population survey

+ Military hours

All divided by non-institutional population 16-64

\section{B.2.2. Sources}

The specific sources of the data listed above are as follows:

○ National accounts, post-1947, quarterly

www.bea.gov, new NIPA Table 1.1.6 (in bil. chained $2000 \$$ )

Gross domestic product (GDP)

Personal consumption expenditures (PCE)

Gross private domestic investment (GPDI)

Net exports of goods and services

Government consumption expenditures and gross investment

www.bea.gov, new NIPA Table 1.1.5 (in bil. \$)

PCE durable goods

PCE nondurable goods

PCE services

www.bea.gov, new NIPA Table 1.1.9 (in $2000=100)$

Deflator, PCE durable goods

Deflator, PCE nondurable goods 
Deflator, PCE services

www.bea.gov, new NIPA Table 3.9 .5 (in bil. \$)

Government consumption expenditures

www.bea.gov, new NIPA Table 3.2 (in bil. \$)

Federal excise taxes

www.bea.gov, new NIPA Table 3.3 (in bil. \$)

State and local sales taxes

State and local other taxes

- Flow of funds accounts, post-1952, quarterly

www.federalreserve.gov, Flow Table 10 (in mil. \$)

Consumption of fixed capital, consumer durables

www.federalreserve.gov, Level Table 100 (in bil. \$)

Current-cost net stock of consumer durables

○ Hours and population, post-1959, quarterly

Prescott, Ueberfeldt, and Cociuba (2005), Hours.xls

Non-institutional hours from the current population survey

Non-institutional population, ages 16-64 


\section{Appendix C. Additional Material Not Reported in Text}

In this Appendix, we describe further details on results that were mentioned only briefly in the main text of the paper.

\section{C.1. Results for Government Consumption Wedge}

Figures A3 and A4 show results with varying government consumption wedges in the benchmark model for the Great Depression and the 1982 recession, respectively. The results for extensions of the benchmark model - with variable capital utilization and with adjustment costs - are almost identical to Figure A3 and therefore not shown.

\section{C.2. Model with Maximum Investment Wedge}

In the paper, we reported on results for the Model with Maximum Investment Wedge. In this case, the investment wedge was chosen to be as large as it needed to be in order for investment in the model and data to line up. In Figure A5, we display the investment series - both model and data-along with consumption and output. (Government consumption in the model is set equal to the 1929 level, but there is little change in the data over the period 1929-1939.) Notice that consumption in the model rises significantly as investment falls implying a consumption anomaly.

\section{C.3. Wedges for the Capital Utilization Model}

Figure A6 shows all wedges for the model with variable capital utilization. In Figure 9 of the paper we displayed only the efficiency wedge. Here, we provide the exact analogue of Figure 1 with all wedges but the government consumption wedge. 


\section{C.4. Results for an Alternative Investment Wedge}

Figure A7 shows that our accounting procedure is not sensitive to choosing $\tau_{k}$ as the investment wedge rather than $\tau_{x}$. This figure shows the model's prediction for output based on the two prototype models described in Sections A.1 and A.2.4. For both, we set the adjustment costs to be extreme $(\eta=1)$ so that we could compare the results to Christiano and Davis (2006).

The line marked Prediction With $\tau_{x}$ Wedge and Extreme Costs in Figure A7 is the same as that in Figure 14 in our paper. The line marked Prediction With $\tau_{k}$ Wedge and Extreme Costs is what Christiano and Davis (2006) would find if they were to apply the same accounting procedure that we do. Here, we use Christiano and Davis' (2006) estimates for the stochastic process, namely,

$$
\begin{aligned}
s_{t+1}=\left[\begin{array}{c}
-.0112 \\
.0232 \\
.00237 \\
-.0150
\end{array}\right]+ & {\left[\begin{array}{cccc}
.952 & .0240 & -0.00000138 & -.00193 \\
.0720 & .924 & .560 & .00263 \\
.00887 & -.00471 & .988 & .000408 \\
-.00448 & .0209 & -.00102 & 0.995
\end{array}\right] s_{t} } \\
& +\left[\begin{array}{cccc}
-.0122 & 0 & 0 & 0 \\
-.000902 & .00659 & 0 & 0 \\
.00235 & .000869 & -.00110 & 0 \\
.000000805 & .00738 & -.00645 & .0134
\end{array}\right] \epsilon_{s, t+1} .
\end{aligned}
$$

In Figure A8, we redisplay the line marked Prediction With $\tau_{k}$ Wedge and Extreme Costs from Figure A7. In Figure A8, we label it Prediction Using a Theoretically-Consistent Methodology. We want to compare this result with the result that Christiano and Davis (2006) actually report, what we refer to as Prediction Using an Alternative Methodology.

As we noted earlier, our propositions distinguish between the direct effect and the forecasting effect of fluctuations in wedges. This turned out not to be quantitatively important for the prototype model with a $\tau_{x}$ investment wedge. ${ }^{6}$ However, it is for the $\tau_{k}$

6 Even so, we redid all of our numerical results applying the accounting procedure that is consistent with our propositions. 
prototype model as Figure A8 shows. In fact, because Christiano and Davis (2006) are not consistently applying the propositions, they find that the investment wedge accounts for one-half of the downfall in output. In fact, this wedge accounts for only about one-fifth of the downfall with the caveat that, even then, adjustment costs have to be extreme.

In summary, our main conclusion - that the investment wedge plays a decidedly tertiary role - is not sensitive to using the alternative prototype model proposed by Christiano and Davis (2006).

\section{C.5. Proof of Propositions}

Here, we provide a proof of Proposition 1. The logic behind the other proofs is the same.

The first-order conditions for the gross output problem are $p_{1 t} q_{1 t}=\gamma q_{t}$ and $p_{2 t} q_{2 t}=$ $(1-\gamma) q_{t}$. The first-order conditions for the sector $i$ gross output problem are $\theta p_{i t} q_{i t} / m_{i t}=$ $1+\tau_{i t}$ and $(1-\theta) p_{i t} q_{i t} / z_{i t}=v_{t}$. Thus, $m_{1 t}=\theta \gamma q_{t} /\left(1+\tau_{1 t}\right)$ and $m_{2 t}=\theta \gamma q_{t} /\left(1+\tau_{2 t}\right)$ so $m_{t}=\theta q_{t}\left[\gamma /\left(1+\tau_{1 t}\right)+(1-\gamma) /\left(1+\tau_{2 t}\right)\right]$. The first-order conditions also imply $z_{1 t}=\gamma z_{t}$, $z_{2 t}=(1-\gamma) z_{t}$, and $v_{t}=(1-\theta) q_{t} / z_{t}$. Hence,

$$
y_{t}=q_{t}-m_{t}=\left[1-\theta\left(a_{1 t}+a_{2 t}\right)\right] q_{t}
$$

and substituting the expressions for $m_{i t}$ and $z_{i t}$ into $q_{t}=\left(m_{1 t}^{\theta} z_{1 t}^{1-\theta}\right)^{\gamma}\left(m_{2 t}^{\theta} z_{2 t}^{1-\theta}\right)^{1-\gamma}$ and manipulating gives

$$
q_{t}=\kappa\left(a_{1 t}^{1-\gamma} a_{2 t}^{\gamma}\right)^{\frac{\theta}{1-\theta}} z_{t}
$$

Combining the expressions for $y_{t}$ and $q_{t}$ and using $z_{t}=F\left(k_{t}, l_{t}\right)$ gives the expression for $A_{t}$.

To verify our expression for $\left(1-\tau_{l t}\right)$, compare the first-order condition $v_{t} F_{l t}=w_{t}$ from the composite goods producer in the detailed economy to the first-order condition $\left(1-\tau_{l t}\right) A_{t} F_{l t}=w_{t}$ in the prototype economy to note that $\left(1-\tau_{l t}\right)=v_{t} / A_{t}$ and use 
$v_{t}=(1-\theta) q_{t} / z,(C .5 .2)$, and the expression for $A_{t}$. The derivation of the expression for $\left(1-\tau_{k t}\right)$ is analogous.

\section{C.6. MLE Estimates for Alternative Models}

In Table A1, we report the parameter estimates for the model with variable capital utilization and annual data over the period 1901-1940. In Tables A2 and A3, we report the estimates for the model with adjustment costs, at the BGG level and four times the BGG level, based on annual data for 1901-1940. The quarterly estimates for the adjustment-cost models are reported in Tables A4 and A5. These estimates are based on quarterly data for the period 1959:1-2004:3.

The hillclimbing procedure that we used had no problems finding maxima (at least locally) with the annual dataset during the 1901-1940 period. Estimation in the postwar period was more difficult, and the hillclimbing procedure oftentimes had difficulty finding higher points on the likelihood surface. Thus, in all cases for the postwar, we initialized guesses using estimates based on annual data for the period 1901-2000, which were converted to quarterly estimates. We also perturbed the parameter estimates (after the hillclimbing routine could not find a higher point) many times in search of higher points. We did not find our results to be sensitive to the estimates of the stochastic process. 


\section{References}

Christiano, Lawrence and Joshua Davis. 2006. Two Flaws in Business Cycle Accounting. Manuscript, Northwestern University.

Federal Reserve Board of Governors. Flow of Funds Accounts. 1945-2005. Washington, DC: Federal Reserve Board. (Contains flow and level tables, the latest of which are available at www.federalreserve.gov.)

Kendrick, John W. 1961. Productivity Trends in the United States. Princeton, NJ: Princeton University Press.

Prescott, Edward C., Alexander Ueberfeldt, and Simona Cociuba. 2005. U.S. Hours and Productivity Behavior Using CPS Hours Worked Data: 1959-I to 2004-IV. Manuscript, Federal Reserve Bank of Minneapolis.

Tauchen, George. 1986. Finite State Markov-Chain Approximations to Univariate and Vector Autoregressions. Economic Letters, 20(2): 177-181.

U.S. Department of Commerce. Bureau of the Census. 1975. Historical Statistics of the United States, Colonial to 1970. Washington, DC: U.S. Government Printing Office.

U.S. Department of Commerce. Bureau of Economic Analysis. 1929-2005. Survey of Current Business. Washington, DC: U.S. Government Printing Office. (Contains NIPA tables and fixed asset tables, the latest of which are available at www.bea.gov.)

U.S. Executive Office. Council of Economic Advisers. 2001. Economic Report of the President. Washington, DC: U.S. Government Printing Office. 
Figure A1. Logarithm of U.S. Efficiency Wedge

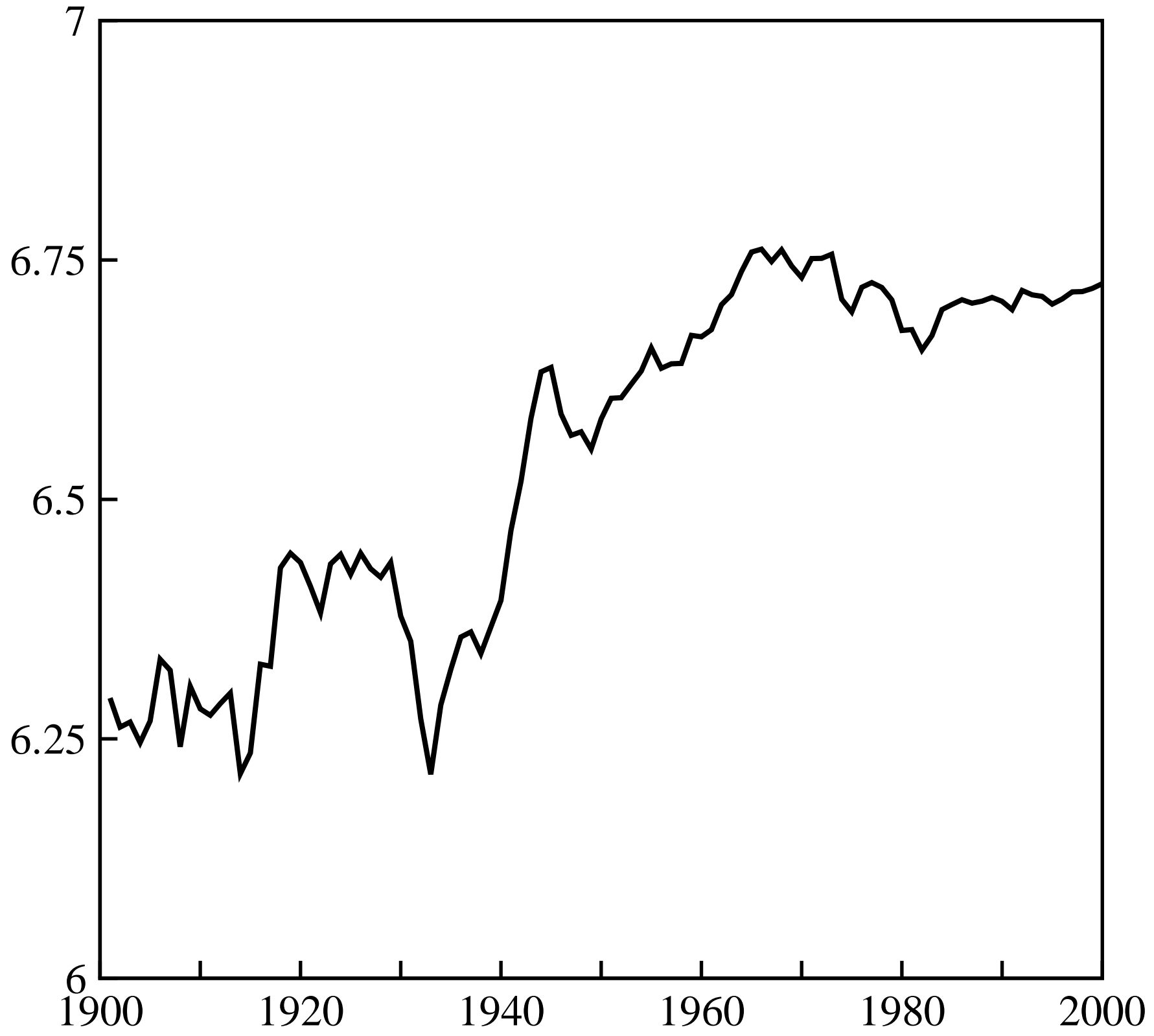


Figure A2. U.S. Labor Wedge

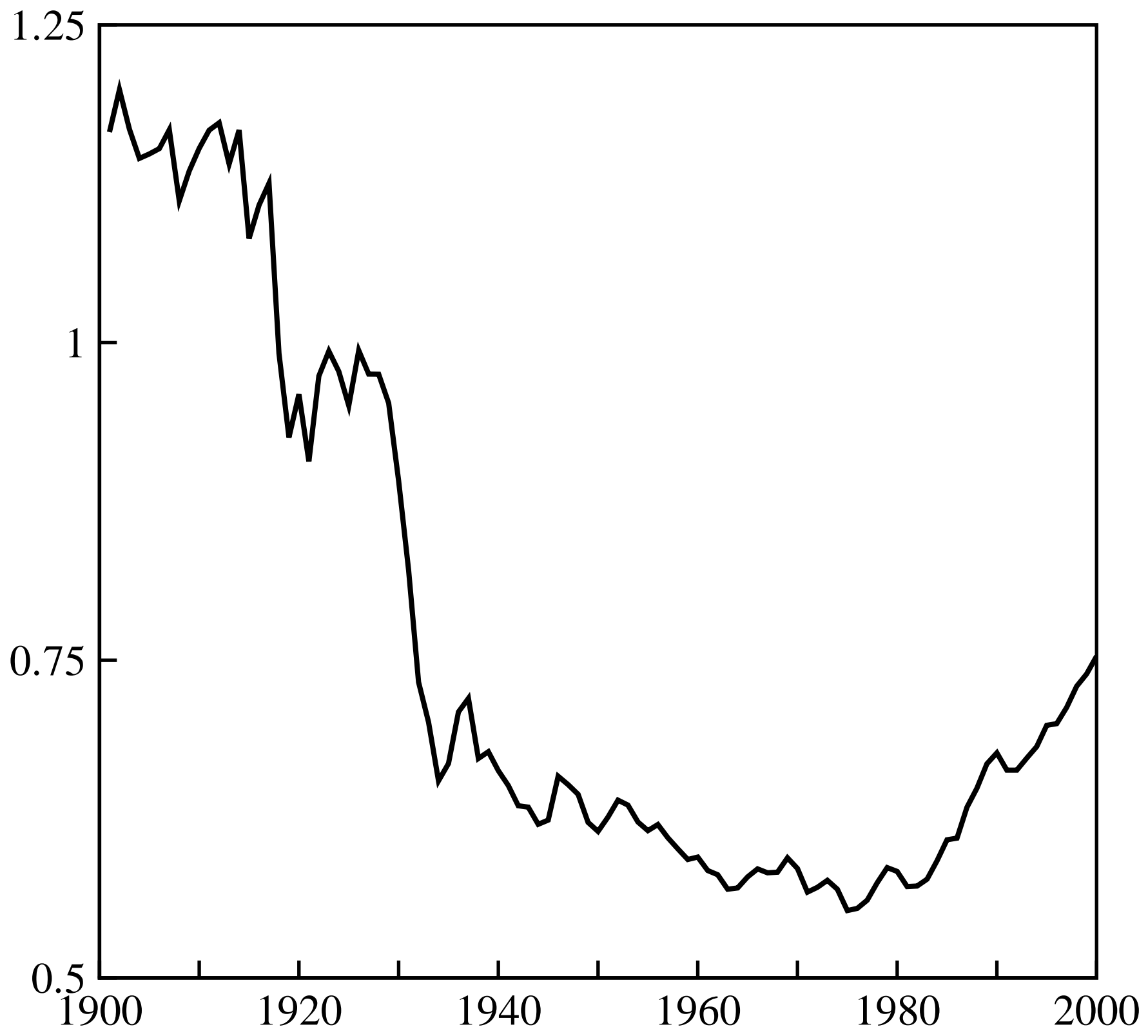


Figure A3

Data and Predictions of Model With Just the Government Consumption Wedge

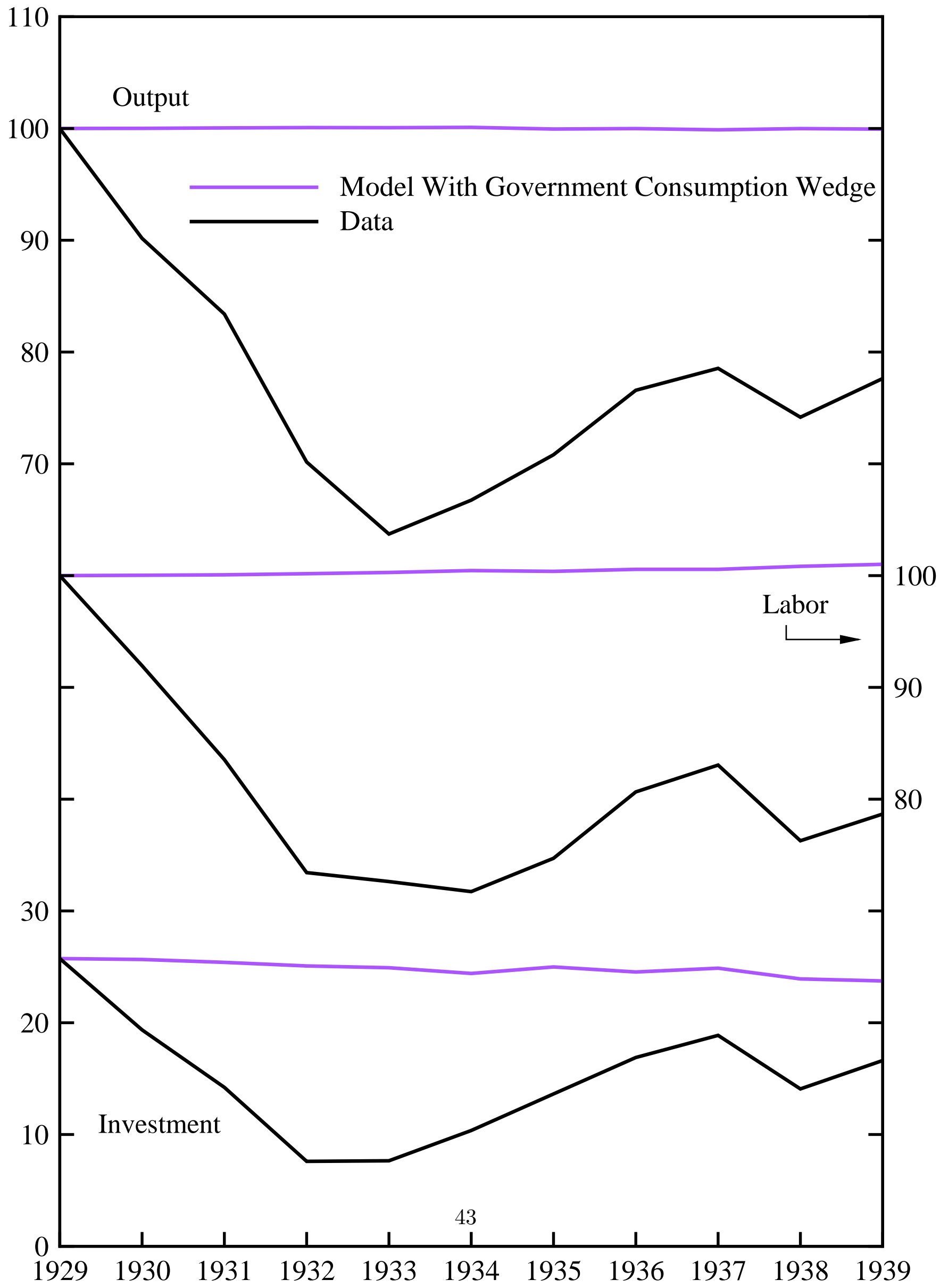


Figure A4

Data and Predictions of Model With Just the Government Consumption Wedge

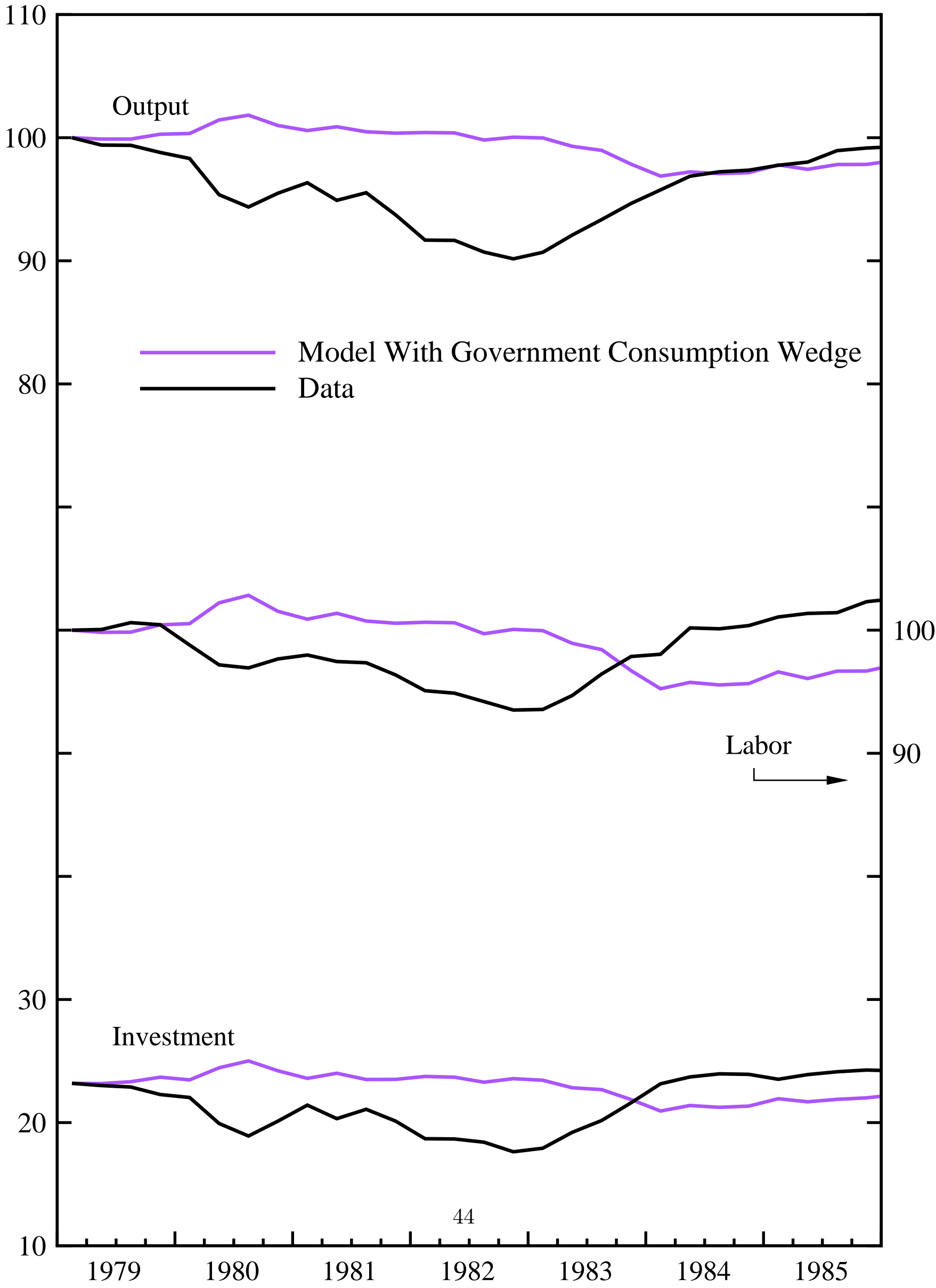


Figure A5

Data and Predictions of Model with Maximum Investment Wedge

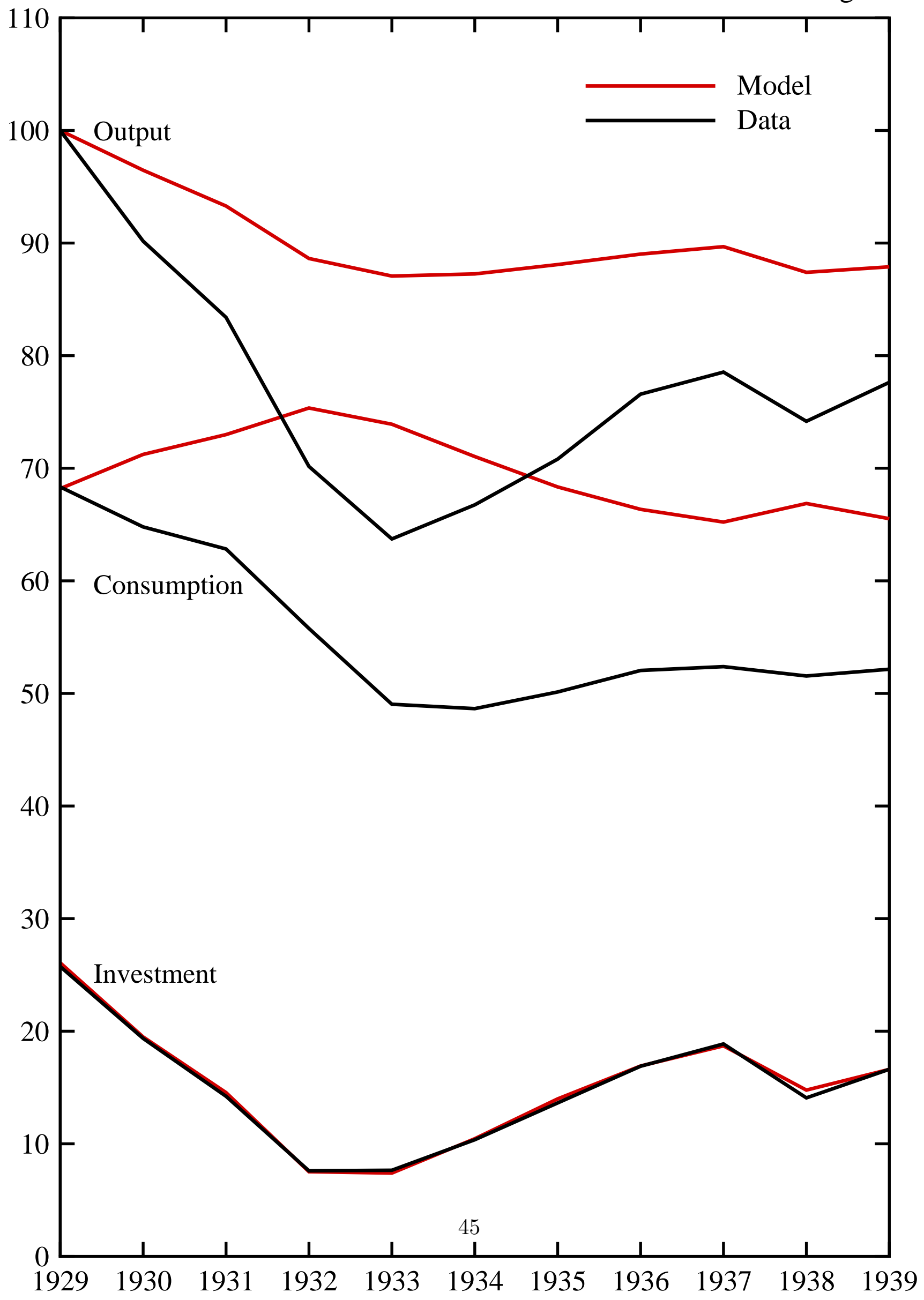


Figure A6

U.S. Output and Three Measured Wedges

for Variable Capital Utilization Specification

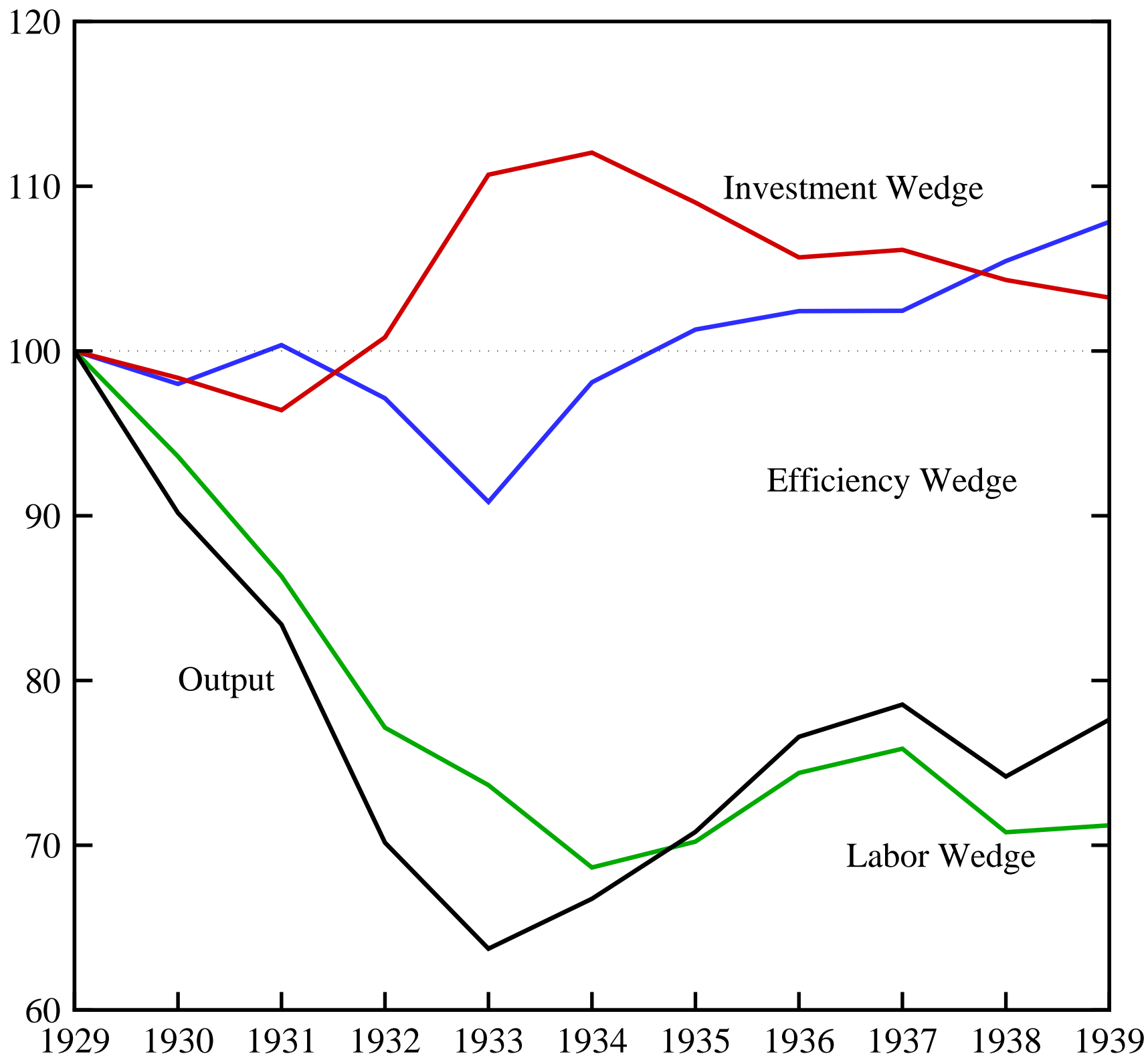


Figure A7

U.S. Output and Predictions of the Models with Just the Investment Wedge and Extreme Adjustment Costs

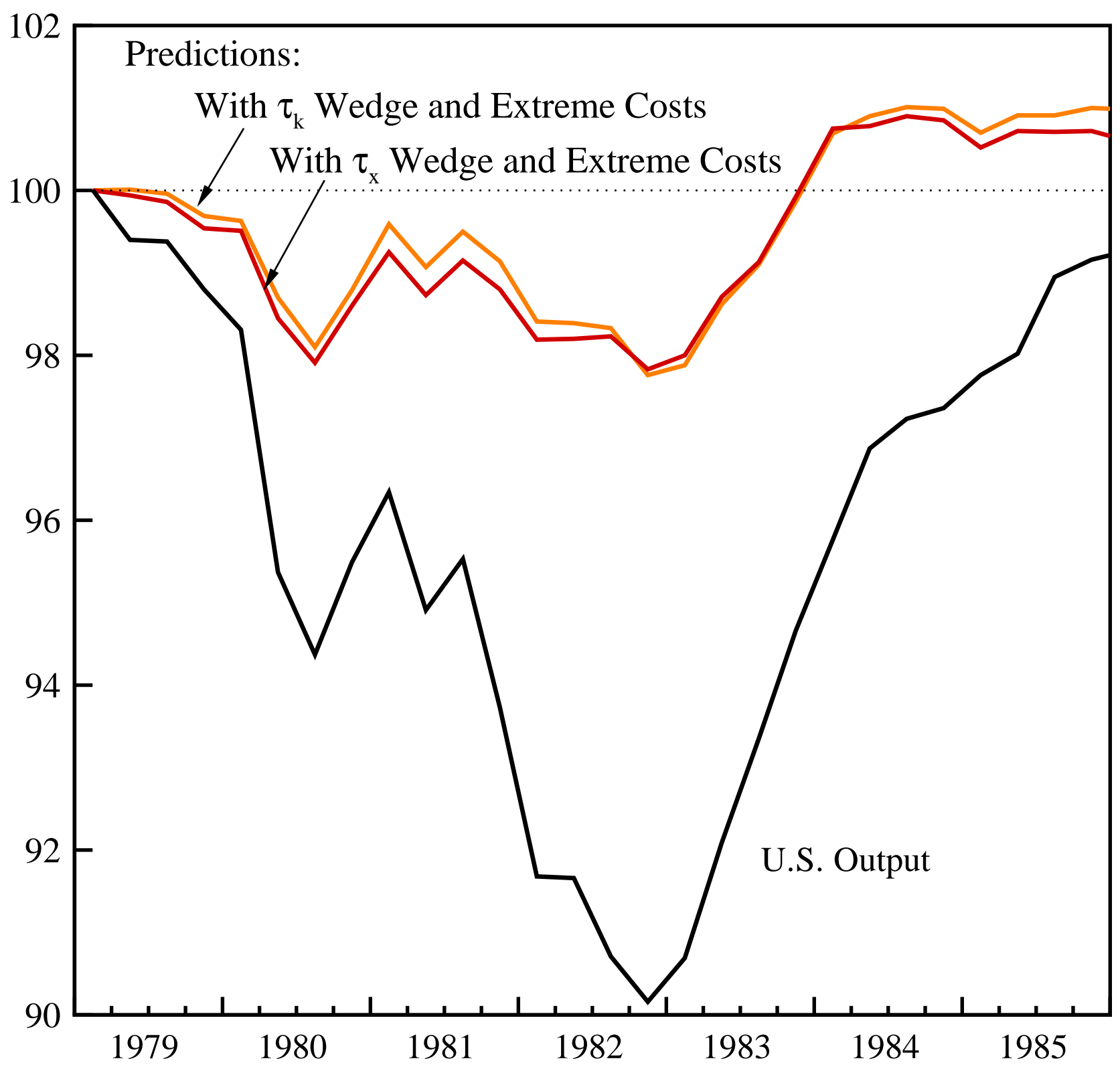


Figure A8

U.S. Output and Predictions of the Model with Just the $\tau_{\mathrm{k}}$ Investment Wedge and Extreme Adjustment Costs

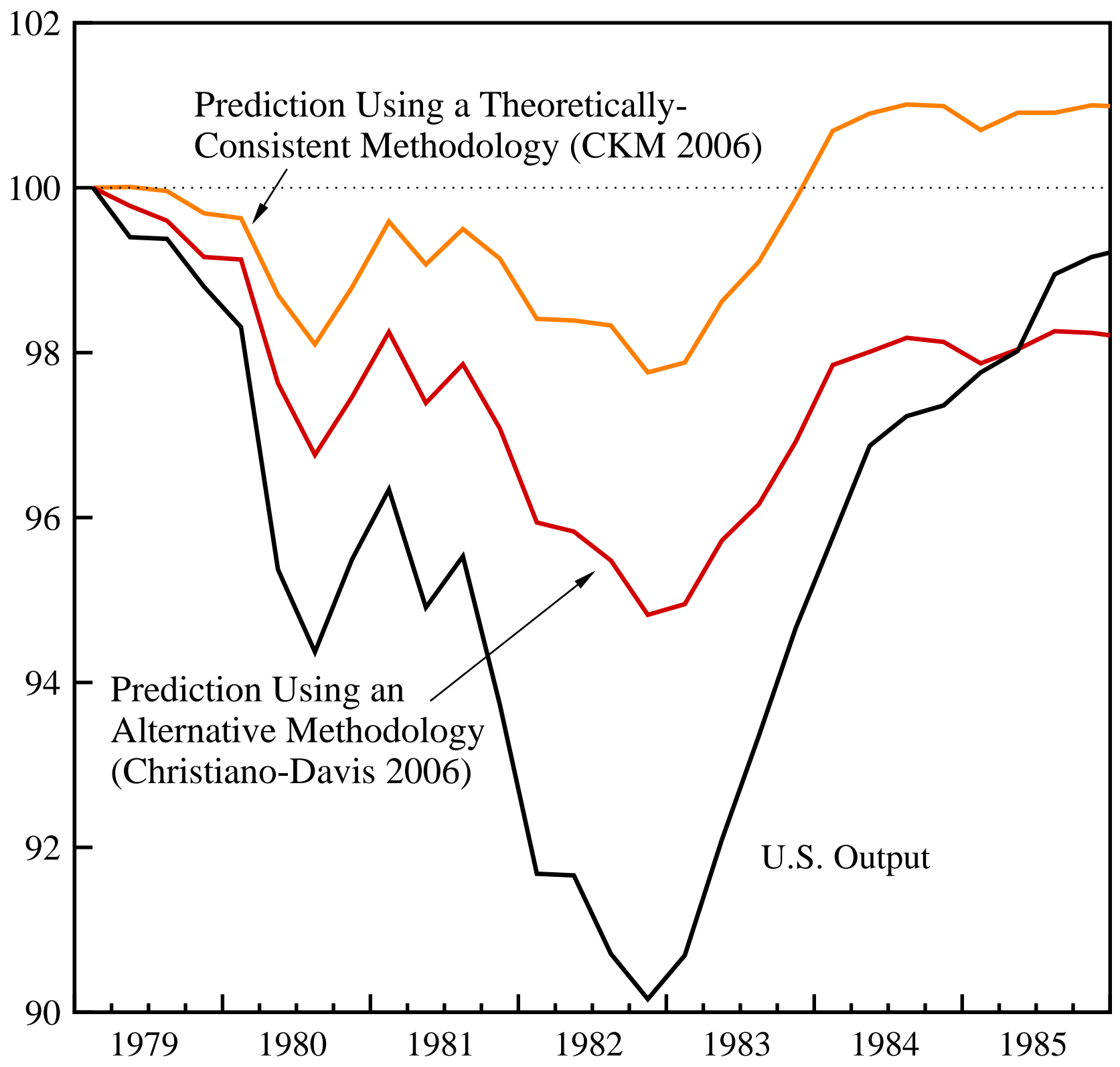


Table A1

Parameters for the Model with Variable Capital Utilization

Annual Data, 1901-1940

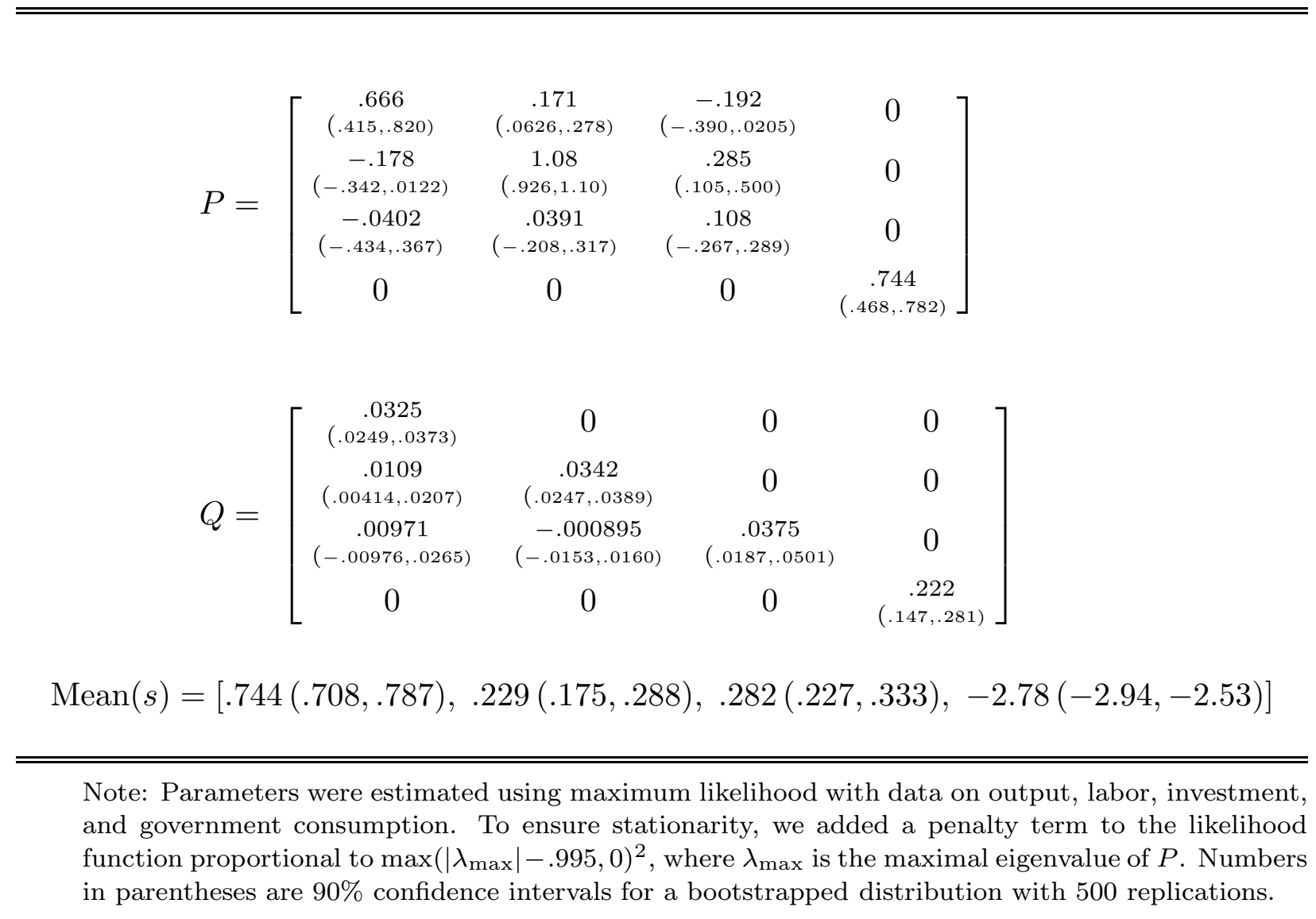




\section{Table A2}

Parameters for the Model with Adjustment Costs at the BGG Level

Annual Data, 1901-1940

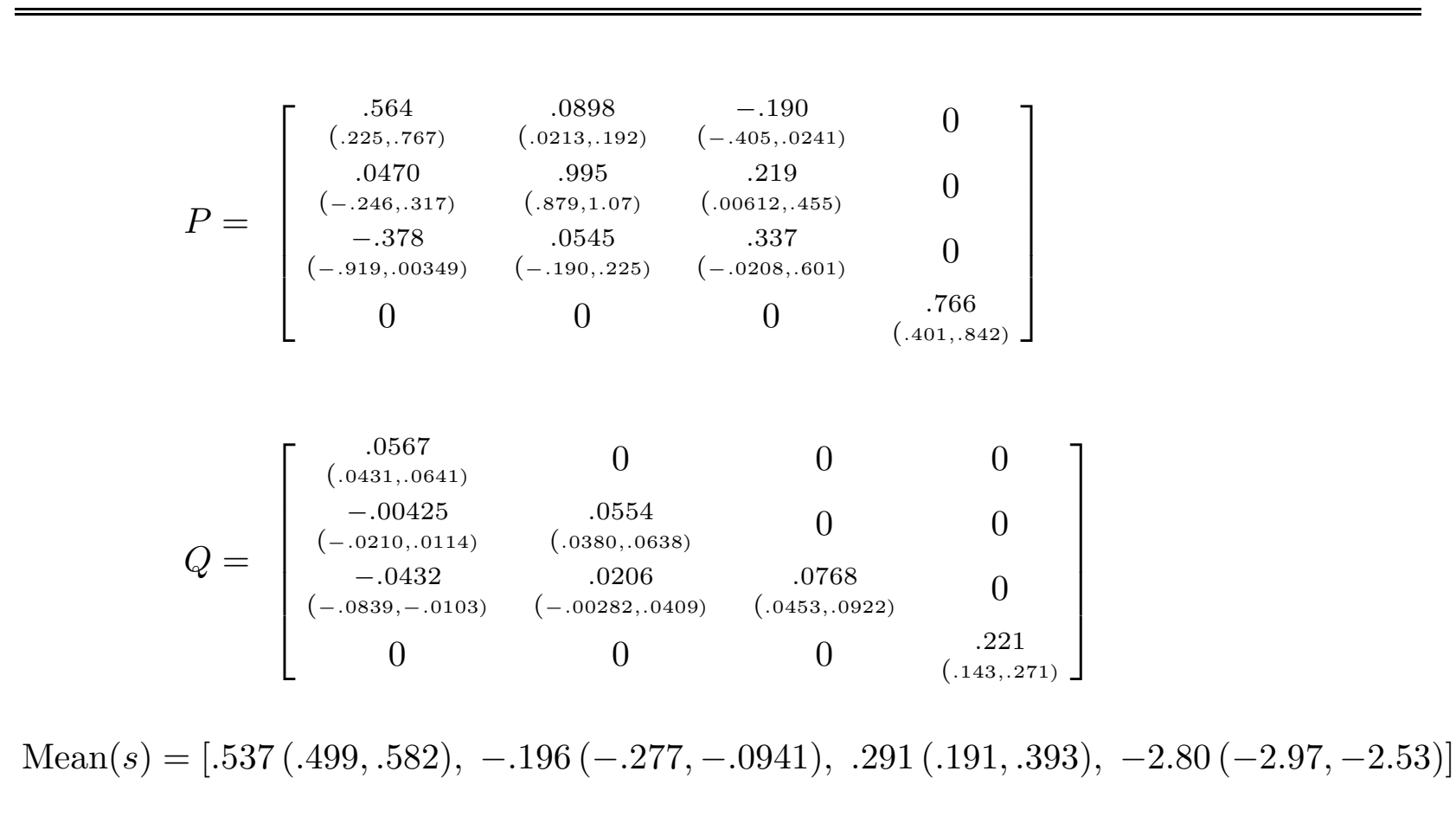

See footnotes to Table A1. 
Table A3

Parameters for the Model with Adjustment Costs at 4 times the BGG Level

Annual Data, 1901-1940

\begin{tabular}{c}
\hline \hline$P=\left[\begin{array}{cccc}.432 & .121 & -.0866 & \\
(-.0446, .625) & (.0476, .276) & (-.191,-.0167) & 0 \\
.178 & .963 & .0938 & 0 \\
(-.160, .603) & (.796,1.03) & (.0169, .196) & 0 \\
-.659 & .112 & .560 & 0 \\
(-1.00,1.00) & (-.419, .331) & (.264, .942) & .783 \\
0 & 0 & 0 & (.433, .874)\end{array}\right]$ \\
$Q=\left[\begin{array}{cccc}.0561 & & & \\
(.0422, .0635) & 0 & 0 & 0 \\
-.00327 & .0553 & 0 & 0 \\
(-.0209, .0143) & (.0381, .0637) & 0 \\
-.176 & .0811 & .189 & 0 \\
(-.252,-.0896) & (.0247, .137) & (.119, .216) & .221 \\
0 & 0 & 0 & (.143, .271)\end{array}\right]$
\end{tabular}

$\operatorname{Mean}(s)=[.536(.497, .577),-.200(-.274,-.0962), .292(.163, .432),-2.81(-3.00,-2.54)]$

See footnotes to Table A1. 
Table A4

Parameters for the Model with Adjustment Costs at the BGG Level

Quarterly Data, 1959:1-2004:3

\begin{tabular}{|c|c|c|c|c|}
\hline \multirow{4}{*}{$P=$} & {$\left[\begin{array}{c}.988 \\
(.988, .988)\end{array}\right.$} & $\begin{array}{c}.0235 \\
(.0232, .0239)\end{array}$ & $\begin{array}{c}.0521 \\
(.0511, .0521)\end{array}$ & $\begin{array}{c}-.0562 \\
(-.0571,-.0562)\end{array}$ \\
\hline & $\begin{array}{c}-.00394 \\
(-.00427,-.00381)\end{array}$ & $\begin{array}{c}.995 \\
(.926,1.10)\end{array}$ & $\begin{array}{c}.0379 \\
(.0374, .0381)\end{array}$ & $\begin{array}{c}-.0297 \\
(-.0303,-.0297)\end{array}$ \\
\hline & $\begin{array}{c}.00739 \\
(.00717, .00744)\end{array}$ & $\begin{array}{c}-.0781 \\
(-.0782,-.0780)\end{array}$ & $\begin{array}{c}.879 \\
(.879, .880)\end{array}$ & $\begin{array}{c}.146 \\
(.145, .147)\end{array}$ \\
\hline & {$\left[\begin{array}{c}-.00155 \\
(-.00190,-.00113)\end{array}\right.$} & $\begin{array}{c}-.0129 \\
(-.0134,-.0128)\end{array}$ & $\begin{array}{c}.0480 \\
(.0474, .0482)\end{array}$ & $\begin{array}{c}.965 \\
(.964, .965)\end{array}$ \\
\hline \multirow{4}{*}{$Q=$} & {$\left[\begin{array}{c}.0114 \\
(.0109, .0120)\end{array}\right.$} & 0 & 0 & 0 \\
\hline & $\begin{array}{c}.00132 \\
(.000723, .00198)\end{array}$ & $\begin{array}{c}.00641 \\
(.00576, .00701)\end{array}$ & 0 & 0 \\
\hline & $\begin{array}{c}-.00925 \\
(-.00987,-.00863)\end{array}$ & $\begin{array}{c}.00264 \\
(.00175, .00325)\end{array}$ & $\begin{array}{c}.0221 \\
(.0213, .0227)\end{array}$ & 0 \\
\hline & $\begin{array}{c}-.000388 \\
-(-.000910, .000252)\end{array}$ & $\begin{array}{c}.00619 \\
(.00540, .00681)\end{array}$ & $\begin{array}{c}.0135 \\
(.0126, .0141)\end{array}$ & $\left.\begin{array}{c}.00698 \\
(.00626, .00762)\end{array}\right]$ \\
\hline
\end{tabular}

See footnotes to Table A1. 
Table A5

Parameters for the Model with Adjustment Costs at 4 times the BGG Level

Quarterly Data, 1959:1-2004:3

\begin{tabular}{|c|c|c|c|c|}
\hline \multirow{4}{*}{$P=$} & {$\left[\begin{array}{c}1.014 \\
(1.014,1.014)\end{array}\right.$} & $\begin{array}{c}-.0444 \\
(-.0444,-.0444)\end{array}$ & $\begin{array}{c}.0361 \\
(.0361, .0361)\end{array}$ & $\left.\begin{array}{c}-.0168 \\
(-.0168,-.0168)\end{array}\right]$ \\
\hline & $\begin{array}{c}.00700 \\
(.00700, .00700)\end{array}$ & $\begin{array}{c}.953 \\
(.953, .953)\end{array}$ & $\begin{array}{c}.0173 \\
(.0173, .0173)\end{array}$ & $\begin{array}{c}.00403 \\
(.00400, .00403)\end{array}$ \\
\hline & & $\begin{array}{c}.100 \\
(.100, .100)\end{array}$ & $\begin{array}{c}.830 \\
(.830, .830)\end{array}$ & $\begin{array}{c}.140 \\
(.140, .140)\end{array}$ \\
\hline & $\begin{array}{c}-.0379 \\
(-.0379,-.0379)\end{array}$ & $\begin{array}{c}-.0158 \\
(-.0158,-.0158)\end{array}$ & $\begin{array}{c}-.0302 \\
(-.0302,-.0302)\end{array}$ & $\begin{array}{c}1.044 \\
(1.044,1.044)\end{array}$ \\
\hline \multirow{4}{*}{$Q=$} & $\begin{array}{c}.0114 \\
(.0114, .0114)\end{array}$ & 0 & 0 & 0 \\
\hline & $\begin{array}{c}.00148 \\
(.00148, .00149)\end{array}$ & $\begin{array}{c}.00626 \\
(.00626, .00627)\end{array}$ & 0 & 0 \\
\hline & $\begin{array}{c}-.0272 \\
(-.0272,-.0272)\end{array}$ & $\begin{array}{c}.0122 \\
(.0122, .0122)\end{array}$ & $\begin{array}{c}.0243 \\
(.0243, .0243)\end{array}$ & 0 \\
\hline & {$\left[\begin{array}{c}-.0000298 \\
(-.0000317,-.0000287\end{array}\right.$} & $\begin{array}{c}.00589 \\
(.00589, .00589)\end{array}$ & $\begin{array}{c}.00929 \\
(.00929, .00929)\end{array}$ & $\begin{array}{c}.0115 \\
(.0115, .0115)\end{array}$ \\
\hline
\end{tabular}

See footnotes to Table A1. 